\title{
FRACTIONAL IDEALS AND INTEGRATION WITH RESPECT TO THE GENERALISED EULER CHARACTERISTIC
}

\author{
JULIO JOSÉ MOYANO-FERNÁNDEZ
}

\begin{abstract}
Let $\mathfrak{b}$ be a fractional ideal of a one-dimensional CohenMacaulay local ring $\mathcal{O}$ containing a perfect field $k$. This paper is devoted to the study some $\mathcal{O}$-modules associated with $\mathfrak{b}$. In addition, different motivic Poincaré series are introduced by considering ideal filtrations associated with $\mathfrak{b}$; the corresponding functional equations of these Poincaré series are also described.
\end{abstract}

\section{INTRODUCTION}

Canonical ideals over one-dimensional Cohen-Macaulay rings were profusely studied by Kunz, Herzog, et al., giving nice characterisations of the Gorenstein property: the formula bringing conductor and deltainvariant together, or relating it with symmetry properties of the value semigroup of the ring (cf. e.g. [10]; 8] ). This idea was developed by Delgado for several branches of complex curve singularities (see [6]), and by Campillo, Delgado and Kiyek in a more general context (see [5]); they also introduced a Poincaré series $P(\underline{t})$, deducing its functional equations when the ring is Gorenstein. Later, Campillo, Delgado and Gusein-Zade showed that this Poincaré series coincides with the integral over the projectivisation of the ring with respect to the Euler characteristic (cf. [2, [3]). In fact, a motivic approach to $P(\underline{t})$ was also introduced just by taking in the integral the generalised Euler characteristic instead. Recently, some connections with number-theoretical local zeta functions were founded by specialising to the case of finite

\footnotetext{
1991 Mathematics Subject Classification. Primary 14H20; Secondary 14G10;13H10.

Key words and phrases. Fractional ideal, Poincaré series, one-dimensional local ring, motivic integration, functional equation.

The author was partially supported by the Spanish Government Ministerio de Educación y Ciencia (MEC) grant MTM2007-64704 in cooperation with the European Union in the framework of the founds "FEDER", and by the Deutsche Forschungsgemeinschaft (DFG).
} 
fields (cf. [7]). In that paper, the authors introduce integrals with respect to the generalised Euler characteristic over fractional ideals of the ring. The aim of the actual work is to discuss more in detail such motivic integrals, which turn out to be Poincaré series of fractional ideals, and related objects; in particular, we extend some of the results concerning the "classical" series $P(\underline{t})$ and the Gorenstein property contained in [8] and [5]. We will also use techniques of motivic integration.

The paper goes as follows. Section 2 is an introduction to the notion of canonical ideal. We define the dual of an ideal, characterise the self-dual ideals and show that it is indeed an extension of well-known properties of Gorenstein rings (Theorem (2.12)). Section 3 is devoted to study an analogous of the value semigroup $S(\mathcal{O})$ of a one-dimensional Cohen-Macaulay local ring $\mathcal{O}$ for a fractional ideal $\mathfrak{b}$ : the resulting set $S(\mathfrak{b})$ is no longer a semigroup, but it has structure of module over $S(\mathcal{O})$. We will give a notion of the symmetry of $S(\mathfrak{b})$; the statements of Section 2 allow us to characterise the symmetry of $S(\mathfrak{b})$ (cf. Proposition (3.8)). In Section 4 we define a Poincaré series of motivic nature for

the fractional ideal $\mathfrak{b}$ (Definition $(4.10)$ ); we prove its rationality and also its functional equations in absence of the Gorenstein condition (Theorem (4.19)). Finally, in Section 5 we investigate the analogous of the extended semigroup $\widehat{S}(\mathcal{O})$ of the ring $\mathcal{O}$ for a fractional ideal-it has again structure of $\widehat{S}(\mathcal{O})$-module--, as well as an alternative motivic Poincaré series associated with $\mathfrak{b}$, giving its functional equations as well (see Proposition (5.5), Theorem (5.8)).

\section{DUALity AND FRACTIONAL IDEALS}

(2.1) Let $\mathcal{O}$ be a one-dimensional Cohen-Macaulay local ring containing a perfect field $k$ with maximal ideal $\mathfrak{m}$. Let $\overline{\mathcal{O}}$ be its integral closure with respect to its total ring of fractions $\mathcal{K}$. Let us assume that $\overline{\mathcal{O}}$ is a finitely generated $\mathcal{O}$-module and that the degree $\rho:=[\mathcal{O} / \mathfrak{m}: k]$ is finite. Let $\delta:=\operatorname{dim}_{k}(\overline{\mathcal{O}} / \mathcal{O})$ be the $\delta$-invariant of the ring $\mathcal{O}$.

The ring $\overline{\mathcal{O}}$ decomposes into a finite intersection of Manis valuation rings, let us say $\overline{\mathcal{O}}=V_{1} \cap \ldots \cap V_{r}$. If $\mathfrak{m}\left(V_{i}\right)$ denotes the maximal ideal of $V_{i}$ for every $i \in\{1, \ldots, r\}$, then the ideals $\mathfrak{m}_{i}:=\mathfrak{m}\left(V_{i}\right) \cap \overline{\mathcal{O}}$ are principal, regular and maximal (cf. [9, Theorem II.(2.11)]) so that $\mathfrak{m}_{i}=t_{i} \overline{\mathcal{O}}$ for every $i \in\{1, \ldots, r\}$. If $k_{i}:=V_{i} / \mathfrak{m}\left(V_{i}\right)=\overline{\mathcal{O}} / \mathfrak{m}_{i}$ for every 
$i \in\{1, \ldots, r\}$, then the extension degrees

$$
d_{i}:=\left[k_{i}: k\right], \quad i \in\{1, \ldots, r\}
$$

are finite (because $\overline{\mathcal{O}}$ is a finitely generated $\mathcal{O}$-module). Let us also define $d:=d_{1}+\ldots+d_{r}$.

(2.2) Recall that a fractional ideal of a $\operatorname{ring} R$ is a $R$-submodule $\mathfrak{a} \neq(0)$ of the total ring of quotients of $R$ such that $a \mathfrak{a} \subseteq R$ for some $a \in \mathcal{O} \backslash\{0\}$. Let us take a fractional ideal $\mathfrak{a}$ of $\overline{\mathcal{O}}$. It is easy to see that $\mathfrak{a}$ can be written uniquely as a product $\mathfrak{a}=\mathfrak{m}_{1}^{v_{1}} \cdot \ldots \cdot \mathfrak{m}_{r}^{v_{r}}$ for $\underline{v}:=\left(v_{1}, \ldots, v_{r}\right) \in$ $\mathbb{Z}^{r}$. We will denote $\mathfrak{m}^{\underline{v}}:=\mathfrak{m}_{1}^{v_{1}} \cdot \ldots \cdot \mathfrak{m}_{r}^{v_{r}}$ and $\underline{v}(\mathfrak{a}):=v_{1}+\ldots+v_{r}$.

(2.3) Definition: A fractional ideal $\mathfrak{c}$ of $\mathcal{O}$ is called canonical if it satisfies the following two properties:

(a) $\mathfrak{c} \cdot \mathcal{K}=\mathcal{K}$ (i.e., $\mathfrak{c}$ is a regular ideal of $\mathcal{O}$ ).

(b) For any regular fractional ideal $\mathfrak{a}$ of $\mathcal{O}$ one has that

$$
\mathfrak{a}=\mathfrak{c}:(\mathfrak{c}: \mathfrak{a})
$$

(2.4) Since $\mathcal{O}$ is a one-dimensional local ring and $\overline{\mathcal{O}}$ is a finitely generated $\mathcal{O}$-module, a canonical ideal of $\mathcal{O}$ does always exist (it is a consequence of [8, Satz 2.9, p. 22] and [8, Korollar 2.12, p. 24]).

(2.5) Let us fix a canonical ideal $\mathfrak{c}$ of $\mathcal{O}$. For every regular fractional ideal $\mathfrak{a}$ of $\mathcal{O}$, we shall denote $\mathfrak{a}^{*}:=(\mathfrak{c}: \mathfrak{a})$. The ideal $\mathfrak{a}^{*}$ will be called the dual (ideal) of $\mathfrak{a}$. The ideal $\mathfrak{a}$ is said to be self-dual if $\mathfrak{a}=\mathfrak{a}^{*}$.

(2.6) Lemma: The ring $\mathcal{O}$ is a canonical ideal of $\mathcal{O}$ if and only if $\mathcal{O}$ is self-dual.

Proof. If $\mathcal{O}$ is canonical, it belongs to the same class (modulo linear equivalence) as the ideal $\mathfrak{c}$. Then $\mathcal{O}^{*}=\mathfrak{c}: \mathcal{O}=\mathcal{O}: \mathcal{O}=\mathcal{O}$. Conversely, assume $\mathcal{O}=\mathfrak{c}: \mathcal{O}$; we have $\mathfrak{c}: \mathfrak{c}=\mathfrak{c}^{*}=\mathcal{O}$ ([8, Bemerkung 2.5, p.19]), therefore $\mathfrak{c}=\mathcal{O}^{*}$.

(2.7) Notice that the conditions of (2.6) are equivalent to the Gorenstein condition (cf. [8, Korollar 3.4, p. 27]). There is also a useful numerical equivalence given by Theorem (2.9).

(2.8) Let us denote by $\lambda_{\mathcal{O}}(\cdot)=\lambda(\cdot)$ the length of a finite $\mathcal{O}$-module. Moreover, we denote $\mathfrak{f}:=(\mathcal{O}: \overline{\mathcal{O}})$; it will be called the conductor ideal of $\mathcal{O}$ in $\overline{\mathcal{O}}$, and we can easily check that it is the biggest ideal of $\mathcal{O}$ and $\overline{\mathcal{O}}$ at the same time. Let us define $\underline{v}(\mathfrak{f})=: \gamma$ and

$$
\gamma^{\mathfrak{b}}:=\underline{v}((\mathfrak{b}: \overline{\mathcal{O}}): \mathfrak{b})=\underline{v}(\mathfrak{b}: \overline{\mathcal{O}})-\underline{v}(\mathfrak{b} \cdot \overline{\mathcal{O}}) .
$$

Notice that $\gamma^{\mathcal{O}}=\gamma$. 
(2.9) Theorem: (Gorenstein; Apéry; Samuel; Herzog, Kunz) We have:

$$
2 \lambda(\mathcal{O} / \mathfrak{f}) \leq \lambda(\overline{\mathcal{O}} / \mathfrak{f})
$$

Moreover, the equality holds if and only if the ring $\mathcal{O}$ is Gorenstein.

(2.10) The rest of the section is devoted to generalise Theorem (2.9) to any fractional ideal of $\mathcal{O}$. The obvious task is to find a candidate to substitute the conductor ideal in the formula preserving such dimensions. It is easy now: the ideal $\mathfrak{b}: \overline{\mathcal{O}}$ is the biggest fractional $\overline{\mathcal{O}}$-ideal in $\mathfrak{b}^{*}$ and the ideal $\mathfrak{b} \cdot \overline{\mathcal{O}}$ is the smallest $\overline{\mathcal{O}}$-ideal containing $\mathfrak{b}$ and $\overline{\mathcal{O}}$. Notice also that $(\mathfrak{b}: \overline{\mathcal{O}})^{*}=\mathfrak{b}^{*} \cdot \overline{\mathcal{O}}$ and $(\mathfrak{b} \cdot \overline{\mathcal{O}})^{*}=\left(\mathfrak{b}^{*}: \overline{\mathcal{O}}\right)$. First of all note the following fact (cf. [12]):

(2.11) Lemma: We have

$$
2 \lambda\left(\mathfrak{b}^{*} \cdot \mathcal{O} / \mathfrak{b}^{*}\right)=2 \lambda(\mathfrak{b} / \mathfrak{b}: \overline{\mathcal{O}}) .
$$

(2.12) Theorem: Let $\mathfrak{b}$ be a fractional ideal of $\mathcal{O}$. We have

$$
2 \lambda(\mathfrak{b} / \mathfrak{b}: \overline{\mathcal{O}}) \leq \lambda(\mathfrak{b} \cdot \overline{\mathcal{O}} / \mathfrak{b}: \overline{\mathcal{O}}) .
$$

Moreover, the equality holds if and only if $\mathfrak{b}$ is self-dual.

Proof. Without loss of generality, let us assume that $\mathfrak{b} \subseteq \mathcal{O} \subseteq \mathfrak{c} \subseteq \overline{\mathcal{O}}$. Now $\mathfrak{b}^{*}=\mathfrak{c}: \mathfrak{b} \supseteq \mathfrak{b}$. Indeed $\mathfrak{b}^{*} \supseteq \mathfrak{c}^{*}=\mathcal{O} \supseteq \mathfrak{b}$. Then we have

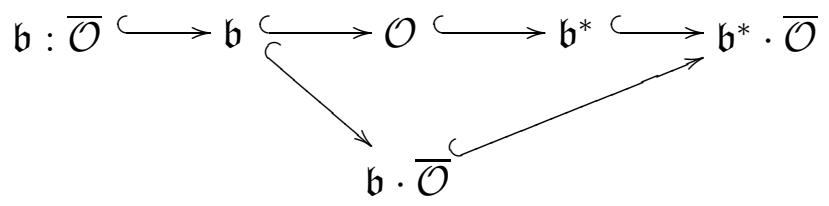

By looking at $(\dagger)$ we have

$$
\begin{aligned}
\lambda\left(\mathfrak{b}^{*} \cdot \overline{\mathcal{O}} / \mathfrak{b}: \overline{\mathcal{O}}\right) & =\lambda\left(\mathfrak{b}^{*} \cdot \overline{\mathcal{O}} / \mathfrak{b} \cdot \overline{\mathcal{O}}\right)+\lambda(\mathfrak{b} \cdot \overline{\mathcal{O}} / \mathfrak{b}: \overline{\mathcal{O}}) \\
& =\lambda\left(\mathfrak{b}^{*} \cdot \overline{\mathcal{O}} / \mathfrak{b} \cdot \overline{\mathcal{O}}\right)+\lambda(\mathfrak{b} \cdot \overline{\mathcal{O}} / \mathfrak{b})+\lambda(\mathfrak{b} / \mathfrak{b}: \overline{\mathcal{O}})
\end{aligned}
$$

If $\mathfrak{b}$ is self-dual, then $\lambda(\mathfrak{b} \cdot \overline{\mathcal{O}} / \mathfrak{b})=\lambda(\mathfrak{b} / \mathfrak{b}: \overline{\mathcal{O}})$ by Lemma $(2.11)$, and by substituting above we are done. Conversely, assuming the following equalities hold:

$$
\lambda(\mathfrak{b} \cdot \overline{\mathcal{O}} / \mathfrak{b}: \overline{\mathcal{O}}) \stackrel{(1)}{=} 2 \lambda(\mathfrak{b} / \mathfrak{b}: \overline{\mathcal{O}}) \stackrel{(2)}{=} 2 \lambda\left(\mathfrak{b}^{*} \cdot \mathcal{O} / \mathfrak{b}^{*}\right)
$$

again looking at $(\dagger)$ we get

$$
\lambda\left(\mathfrak{b}^{*} \cdot \overline{\mathcal{O}} / \mathfrak{b} \cdot \overline{\mathcal{O}}\right) \stackrel{(3)}{=} \lambda\left(\mathfrak{b}^{*} \cdot \overline{\mathcal{O}} / \mathfrak{b}^{*}\right)+\lambda\left(\mathfrak{b}^{*} / \mathfrak{b}\right)+\lambda(\mathfrak{b} / \mathfrak{b}: \overline{\mathcal{O}})
$$


By (1) and (2)

$\lambda\left(\mathfrak{b}^{*} \cdot \overline{\mathcal{O}} / \mathfrak{b} \cdot \overline{\mathcal{O}}\right)=\lambda(\mathfrak{b} / \mathfrak{b}: \overline{\mathcal{O}})+\lambda(\mathfrak{b} / \mathfrak{b}: \overline{\mathcal{O}})=\lambda(\mathfrak{b} / \mathfrak{b}: \overline{\mathcal{O}})+\lambda\left(\mathfrak{b}^{*} \cdot \mathcal{O} / \mathfrak{b}^{*}\right)$,

and plugging the last equality into (3) shows $\lambda\left(\mathfrak{b}^{*} / \mathfrak{b}\right)=0$, i.e., $\mathfrak{b}^{*}=\mathfrak{b}$, hence the ideal $\mathfrak{b}$ is self-dual.

(2.13) Remark: From Lemma (2.11) and Theorem (2.12) it follows: The ideal $\mathfrak{b}$ is self-dual if and only if

$$
2 \lambda(\mathfrak{b} \cdot \mathcal{O} / \mathfrak{b})=2 \lambda(\mathfrak{b} / \mathfrak{b}: \overline{\mathcal{O}})=\lambda(\mathfrak{b} \cdot \overline{\mathcal{O}} / \mathfrak{b}: \overline{\mathcal{O}}) .
$$

(2.14) Let $\underline{v}:=\left(v_{1}, \ldots, v_{r}\right), \underline{w}:=\left(w_{1}, \ldots, w_{r}\right)$ be vectors in $\mathbb{Z}^{r}$. We will write $\underline{v} \geq \underline{w}$ if and only if $v_{i} \geq w_{i}$ for every $i \in\{1, \ldots, r\}, \underline{0}:=$ $(0,0, \ldots, 0) \in \mathbb{Z}^{r}$ and $\underline{1}:=(1,1, \ldots, 1) \in \mathbb{Z}^{r}$. Moreover, for every subset $I \subseteq I_{0}:=\{1, \ldots, r\}$ let $\sharp I$ be the number of elements in $I$, and let $\underline{1}_{I}$ be the element of $\mathbb{Z}^{r}$ whose $i$ th component is equal to 1 or 0 if $i \in I$ or $i \notin I$ respectively. For any $\underline{v} \in \mathbb{Z}^{r}$ and any fractional ideal $\mathfrak{b}$ in $\mathcal{O}$, we define the set

$$
J^{\mathfrak{b}}(\underline{v}):=\{z \in \mathfrak{b} \backslash\{0\} \mid \underline{v}(z) \geq \underline{v}\},
$$

with $\underline{v}(z):=\left(v_{1}(z), \ldots, v_{r}(z)\right)$. They are ideals defining a multi-index filtration $\left\{J^{\mathfrak{b}}(\underline{v})\right\}$, as $J^{\mathfrak{b}}(\underline{v}) \supseteq J^{\mathfrak{b}}(\underline{w})$ if $\underline{w} \geq \underline{v}$.

For every $i \in\{1, \ldots, r\}$, let us define $C^{\mathfrak{b}}(\underline{v}, i):=J^{\mathfrak{b}}(\underline{v}) / J^{\mathfrak{b}}\left(\underline{v}+\underline{1}_{\{i\}}\right)$, and $c^{\mathfrak{b}}(\underline{v}, i):=\operatorname{dim}_{k}\left(C^{\mathfrak{b}}(\underline{v}, i)\right)$; we write also $C^{\mathfrak{b}}(\underline{v}):=J^{\mathfrak{b}}(\underline{v}) / J^{\mathfrak{b}}(\underline{v}+\underline{1})$, as well as $c^{\mathfrak{b}}(\underline{v}):=\operatorname{dim}_{k}\left(C^{\mathfrak{b}}(\underline{v})\right)$. Since $\mathcal{O}$ is Cohen-Macaulay, $c^{\mathfrak{b}}(\underline{v})<\infty$ for every $\underline{v} \in \mathbb{Z}^{r}$; also the filtration is finitely determined, i.e., for any $\underline{v} \in \mathbb{Z}^{r}$ there exists $N \in \mathbb{Z}$ such that $J^{\mathfrak{b}}(\underline{v}) \supset \mathfrak{m}^{N}$; that means that every subspace $J^{\mathfrak{b}}(\underline{v})$ of $\mathcal{O}$ has finite codimension $\ell^{\mathfrak{b}}(\underline{v})$ (cf. [4, p. 194]). Notice that, for every $i \in\{1, \ldots, r\}$ one has $0 \leq c^{\mathfrak{b}}(\underline{v}, i) \leq d_{i}$, and if $\underline{v} \geq \gamma^{\mathfrak{b}}$, then $c^{\mathfrak{b}}(\underline{v}, i)=d_{i}$ for every $i \in\{1, \ldots, r\}$ (The proof of these facts follows much more [5]).

(2.15) The Gorenstein condition on the ring $\mathcal{O}$ was proven to be equivalent to the following equality (cf. [5, Corollary (3.7)]):

$$
c^{\mathcal{O}}(\underline{v})+c^{\mathcal{O}}(\gamma-\underline{v}-\underline{1})=d, \text { for every } \underline{v} \in \mathbb{Z}^{r} .
$$

Our purpose now is to state the analogue of this result for the case of a fractional ideal. The proof is adapted from [5]. We show first:

(2.16) Lemma: We have $c^{\mathfrak{b}}\left(\gamma^{\mathfrak{b}}-\underline{1}_{\{i\}}, i\right)<d_{i}$.

Proof. Write $\gamma^{\mathfrak{b}}=\left(\gamma_{1}^{\mathfrak{b}}, \ldots, \gamma_{r}^{\mathfrak{b}}\right)$. Let $i \in\{1, \ldots, r\}$. Consider the $k$ linear map $\phi_{i}: C^{\mathfrak{b}}\left(\gamma^{\mathfrak{b}}-\underline{1}_{\{i\}}\right) \rightarrow \mathfrak{b} \cdot \overline{\mathcal{O}} / \mathfrak{b}: \overline{\mathcal{O}}$ given by

$$
z \bmod J^{\mathfrak{b}}\left(\gamma^{\mathfrak{b}}-\underline{1}_{\{i\}}\right) \mapsto z \cdot t_{i}^{-\left(\gamma_{i}^{\mathfrak{b}}-1\right)} \bmod \mathfrak{b}: \overline{\mathcal{O}}
$$


This map is clearly injective, so we have to prove that $\phi$ is not an epimorphism. Let $i \in\{1, \ldots, r\}$. It is easily seen that

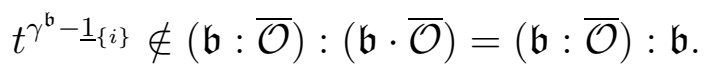

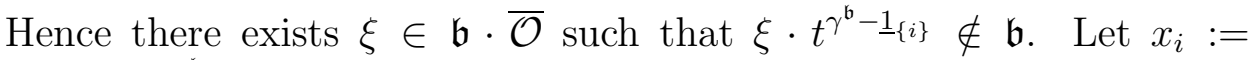
$\xi \cdot t^{\gamma^{\mathfrak{b}}} \cdot t_{i}^{-\gamma_{i}^{\mathfrak{b}}}$. Notice that $x_{i} \in \mathfrak{b} \cdot \overline{\mathcal{O}}$. Furthermore, if $z \in J^{\mathfrak{b}}\left(\gamma^{\mathfrak{b}}-\underline{1}_{\{i\}}\right)$

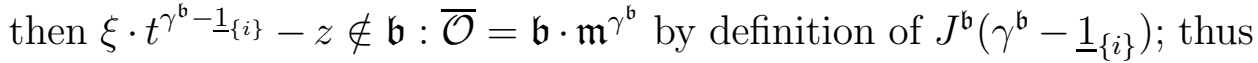

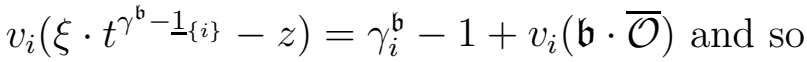

$$
v_{i}\left(x_{i}-\xi \cdot t_{i}^{-\left(\gamma_{i}^{\mathfrak{b}}-1\right)}\right)=v_{i}\left(\xi \cdot t^{\gamma^{\mathfrak{b}}-\underline{1}_{\{i\}}} \cdot t_{i}^{-\gamma_{i}^{\mathfrak{b}}}-z \cdot t_{i}^{1-\gamma_{i}^{\mathfrak{b}}}\right)=v_{i}(\mathfrak{b} \cdot \overline{\mathcal{O}}),
$$

which proves the non-surjectivity of $\phi_{i}$.

(2.17) Proposition: Assume $\mathcal{O}$ to be analytically reduced. Let $\mathfrak{b}$ be a fractional ideal, let $\underline{v} \in \mathbb{Z}^{r}$; then

$$
c^{\mathfrak{b}}(\underline{v}, i)+c^{\mathfrak{b}}\left(\gamma^{\mathfrak{b}}-\underline{v}-\underline{1}_{\{i\}}, i\right) \leq d_{i} \text { for every } i \in\{1, \ldots, r\} .
$$

Proof. For every $\underline{v} \in \mathbb{Z}^{r}$ and every $i \in\{1, \ldots, r\}$, the map $\eta_{\underline{v}, i}$ : $C^{\mathfrak{b}}(\underline{v}, i) \rightarrow k_{i}$ defined by $z \bmod J^{\mathfrak{b}}\left(\underline{v}+\underline{1}_{\{i\}}\right) \mapsto z t_{i}^{-v_{i}} \bmod \mathfrak{m}_{i}$ is a $k$ monomorphism, hence $c^{\mathfrak{b}}(\underline{v}, i) \leq d_{i}$. Take now $\underline{w}=\left(w_{1}, \ldots, w_{r}\right) \in \mathbb{Z}^{r}$ with $\underline{v} \leq \underline{w}$. Thus for every $i \in\{1, \ldots, r\}$, the inclusion $J^{\mathfrak{b}}(\underline{w}) \rightarrow$ $J^{\mathfrak{b}}(\underline{v})$ induces a $k$-homomorphism $\varphi_{\underline{w}, \underline{v}, i}: C^{\mathfrak{b}}(\underline{w}, i) \rightarrow C^{\mathfrak{b}}(\underline{v}, i)$. For an $i \in\{1, \ldots, r\}$ with $v_{i}=w_{i}$ we have $\eta_{\underline{w}, i}=\eta_{\underline{v}, i} \circ \varphi_{\underline{w}, \underline{v}, i}$ and $\varphi_{\underline{w}, \underline{v}, i}$ is injective. Notice also that for every $\underline{n}, \underline{m} \in \mathbb{Z}^{r}$ the following inclusion holds:

$$
J^{\mathfrak{b}}(\underline{v}) J^{\mathcal{O}}(\underline{w})+J^{\mathfrak{b}}(\underline{w}) J^{\mathcal{O}}(\underline{v}) \subseteq J^{\mathfrak{b}}(\underline{v}+\underline{w}) .
$$

Then for $a \in J^{\mathfrak{b}}(\underline{v}), b \in J^{\mathfrak{b}}(\underline{w}), i \in\{1, \ldots, r\}$ we have

$\eta_{\underline{v}, i}\left(a \bmod J^{\mathfrak{b}}\left(\underline{v}+\underline{1}_{\{i\}}\right)\right) \cdot \eta_{\underline{w}, i}\left(b \bmod J^{\mathfrak{b}}\left(\underline{w}+\underline{1}_{\{i\}}\right)\right)=\eta_{\underline{v}+\underline{w}, i}\left(J^{\mathfrak{b}}(a b \bmod \underline{v}+\underline{w})\right)$.

Let $i \in\{1, \ldots, r\}$. Let $H_{i} \subset k_{i}$ be a 1-codimensional subspace of the $k$-vector space $k_{i}$ containing $\operatorname{im}\left(\eta_{\gamma^{\mathfrak{b}}-\underline{1}_{\{i\}}, i}\right)$ (notice that it is possible by Lemma (2.16)); consider the $k$-bilinear pairing $k_{i} \times k_{i} \rightarrow k_{i} \rightarrow k_{i} / H_{i}$ defined by $(a, b) \rightarrow a \cdot b \bmod H_{i}$ (cf. [5. (3.5)]), which is non-degenerate (multiplying by scalars of $k_{i}$ is a $k$-automorphism on $k_{i}$ ). Because of (*) we have

$$
J^{\mathfrak{b}}(\underline{v}) J^{\mathcal{O}}\left(\gamma^{\mathfrak{b}}-\underline{v}-\underline{1}_{\{i\}}\right)+J^{\mathfrak{b}}\left(\gamma^{\mathfrak{b}}-\underline{v}-\underline{1}_{\{i\}}\right) J^{\mathcal{O}}(\underline{v}) \subset J^{\mathfrak{b}}\left(\gamma^{\mathfrak{b}}-\underline{1}_{\{i\}}\right),
$$

therefore $\operatorname{im}\left(\eta_{\underline{v}, i}\right)$ lies in the orthogonal complement of $\operatorname{im}\left(\eta_{\gamma^{\mathfrak{b}}-\underline{v}-\underline{1}_{\{i\}}, i}\right)$, hence

$$
c^{\mathfrak{b}}(\underline{v}, i) \leq d_{i}-c^{\mathfrak{b}}\left(\gamma^{\mathfrak{b}}-\underline{v}-\underline{1}_{\{i\}}, i\right) .
$$


It remains to show that the equality of Proposition (2.17) holds if and only if $\mathfrak{b}$ is self-dual. This follows by the same method as in the proof of [5, Theorem (3.6)], just by applying the characterisation of the self-dual fractional ideals provided by Theorem (2.12) instead of using Theorem $(2.9)$.

(2.18) Lemma: Let $\mathfrak{b} \cdot \overline{\mathcal{O}}=\overline{\mathcal{O}}$. Let $\left\{v^{(p)}\right\}_{0 \leq p \leq h}$ be a strictly increasing sequence in $\mathbb{Z}^{r}$ such that $v^{(0)}=0, v^{(h)}=\gamma^{\mathfrak{b}}$, and for every $p \in$ $\{1, \ldots, h\}$ there exists $i(p) \in\{1, \ldots, r\}$ satisfying $v^{(p)}-v^{(p-1)}=\underline{1}_{\{i(p)\}}$. Then $\mathfrak{b}$ is self-dual if and only if

$$
c^{\mathfrak{b}}\left(v^{(p)}, i(p+1)\right)+c^{\mathfrak{b}}\left(\gamma^{\mathfrak{b}}-v^{(p)}-\underline{1}_{\{i(p+1)\}}, i(p+1)\right)=d_{i(p+1)}
$$

for every $p \in\{0, \ldots, h-1\}$.

Proof. Define $w^{(p)}:=\gamma^{\beta}-v^{(p)}$ for every $p \in\{0, \ldots, h\}$. We have $v^{(p)}+w^{(p+1)}=\gamma^{\mathfrak{b}}-\underline{1}_{\{i(p+1)\}}, w^{(p)}+w^{(p+1)}=\underline{1}_{\{i(p+1)\}}$ and

$$
\begin{gathered}
\mathfrak{b}=J^{\mathfrak{b}}\left(v^{(0)}\right) \supset J^{\mathfrak{b}}\left(v^{(1)}\right) \supset \ldots \supset J^{\mathfrak{b}}\left(v^{(h)}\right)=\mathfrak{b}: \overline{\mathcal{O}} . \\
\mathfrak{b}: \overline{\mathcal{O}}=J^{\mathfrak{b}}\left(w^{(0)}\right) \subset J^{\mathfrak{b}}\left(w^{(1)}\right) \subset \ldots \subset J^{\mathfrak{b}}\left(w^{(h)}\right)=\mathfrak{b} .
\end{gathered}
$$

Therefore

$$
\sum_{p=0}^{h-1} c^{\mathfrak{b}}\left(v^{(p)}, i(p+1)\right)=\operatorname{dim}_{k}(\mathfrak{b} / \mathfrak{b}: \overline{\mathcal{O}})=\sum_{p=0}^{h-1} c^{\mathfrak{b}}\left(w^{(p)}, i(p+1)\right),
$$

and then

$$
2 \operatorname{dim}_{k}(\mathfrak{b} / \mathfrak{b}: \overline{\mathcal{O}})=\sum_{p=0}^{h-1} c^{\mathfrak{b}}\left(v^{(p)}, i(p+1)\right)+c^{\mathfrak{b}}\left(w^{(p)}-\underline{1}_{\{i(p+1)\}}, i(p+1)\right) .
$$

By Proposition (2.17) this expression is smaller than or equal to

$$
\sum_{p=0}^{h-1} d_{i(p+1)}=\sum_{p=1}^{r} \gamma_{i}^{\mathfrak{b}} d_{i}=\operatorname{dim}_{k}(\overline{\mathcal{O}} / \mathfrak{b}: \overline{\mathcal{O}})
$$

and by Theorem (2.12), and the assumption $\mathfrak{b} \cdot \overline{\mathcal{O}}=\overline{\mathcal{O}}$, we are done. The converse follows in the same manner as in the proof of [5, (3.6)], part (d).

(2.19) Theorem: Let $\mathfrak{b}$ be a fractional $\mathcal{O}$-ideal such that $\mathfrak{b} \cdot \overline{\mathcal{O}}=\overline{\mathcal{O}}$. The following statements are equivalent:

(1) For every $\underline{v} \in \mathbb{Z}^{r}$ we have $c^{\mathfrak{b}}(\underline{v}, i)+c^{\mathfrak{b}}\left(\gamma^{\mathfrak{b}}-\underline{v}-\underline{1}_{\{i\}}, i\right) \stackrel{*}{=} d_{i}$, for $i \in\{1, \ldots, r\}$.

(2) $\mathfrak{b}$ is self-dual. 
Proof. If the equality $*$ holds for every $\underline{v} \in \mathbb{Z}^{r}$ and for every $i \in$ $\{1, \ldots, r\}$, then one can choose a strictly increasing sequence as in Lemma (2.18), and we obtain that $2 \operatorname{dim}(\mathfrak{b} / \mathfrak{b}: \overline{\mathcal{O}})=\operatorname{dim}(\overline{\mathcal{O}} / \mathfrak{b}: \overline{\mathcal{O}})$, which by Theorem (2.17) implies the statement.

Analogously as in [5, (3.7)] one shows:

(2.20) Corollary: Let $\underline{v} \in \mathbb{Z}^{r}$. Then

$$
c^{\mathfrak{b}}(\underline{v})+c^{\mathfrak{b}}\left(\gamma^{\mathfrak{b}}-\underline{v}-\underline{1}\right) \leq d=\sum_{i=1}^{r} d_{i} .
$$

Moreover, $\mathfrak{b}$ is self-dual if and only if the equality holds for every $\underline{v} \in \mathbb{Z}^{r}$.

\section{The VAlue ideal of A FRACTiOnAL IDEAL}

Let $\mathfrak{b}$ be a fractional ideal of $\mathcal{O}$. Consider the set

$$
S(\mathfrak{b})=\{\underline{v}(z)-\underline{v}(\mathfrak{b} \cdot \overline{\mathcal{O}}) \mid z \in \mathfrak{b} \backslash\{0\}\} .
$$

This is a subsemigroup of $\mathbb{Z}^{r}$ which is in fact a $S(\mathcal{O})$-module and only depends on the ideal class of $\mathfrak{b}$ in the ideal class semigroup of $\mathcal{O}$. The elements of $S(\mathfrak{b})$ are connected to filtrations $\left\{J^{\mathfrak{b}}(\underline{v})\right\}$ in the following sense:

(3.1) Lemma: Let $k$ be an infinite field. Let $\underline{v}=\left(v_{1}, \ldots, v_{r}\right) \in \mathbb{Z}^{r}$. Then $\underline{v} \in S(\mathfrak{b})$ if and only if $J^{\mathfrak{b}}(\underline{v}) / J^{\mathfrak{b}}\left(\underline{v}+\underline{1}_{\{i\}}\right) \neq 0$ for every $i \in$ $\{1, \ldots, r\}$.

Proof. If $\underline{v} \in S(\mathfrak{b})$, then there exists $\underline{w}=\left(w_{1}, \ldots, w_{r}\right) \in S(\mathfrak{b})$ such that $w_{i}=v_{i}$ and $w_{j} \geq v_{j}$ for every $j \in\{1, \ldots, r\}, j \neq i$. Thus $J^{\mathfrak{b}}(\underline{v}) / J^{\mathfrak{b}}\left(\underline{v}+\underline{1}_{\{i\}}\right) \neq 0$ for every $i \in\{1, \ldots, r\}$. Conversely, assume that $J^{\mathfrak{b}}(\underline{v}) / J^{\mathfrak{b}}\left(\underline{v}+\underline{1}_{\{i\}}\right) \neq 0$ for every $i \in\{1, \ldots, r\}$. For every $i \in\{1, \ldots, r\}$ choose an element $z_{i} \in J^{\mathfrak{b}}(\underline{v}) \backslash J^{\mathfrak{b}}\left(\underline{v}+\underline{1}_{\{i\}}\right)$. Since $k$ is infinite and $k=k_{i}$ for every $i \in\{1, \ldots, r\}$, then there exist elements $a_{1}, \ldots, a_{r} \in$ $\mathcal{O}$ such that $v_{i}\left(a_{1} z_{1}+\ldots+a_{r} z_{r}\right)=v_{i}$ for every $i \in\{1, \ldots, r\}$, i.e., $\underline{v}\left(a_{1} z_{1}+\ldots+a_{r} z_{r}\right)=\underline{v}$.

(3.2) Notice that if $\mathfrak{b}=\mathcal{O}$ then $S(\mathcal{O})$ is the value semigroup of the ring $\mathcal{O}$. Recall that $\gamma^{\mathfrak{b}}:=\underline{v}((\mathfrak{b}: \overline{\mathcal{O}}): \mathfrak{b})=\underline{v}(\mathfrak{b}: \overline{\mathcal{O}})-\underline{v}(\mathfrak{b} \cdot \overline{\mathcal{O}})$, and $\gamma^{\mathcal{O}}=\gamma$ if $\mathfrak{b}=\mathcal{O}$. The next two lemmas show that $\gamma^{\mathfrak{b}}$ plays the role of a kind of conductor of the set $S(\mathfrak{b})$.

(3.3) Lemma: For every fractional ideal $\mathfrak{b}$ in $\mathcal{O}$, there exists $\underline{m} \in \mathbb{Z}^{r}$ such that $J^{\mathcal{K}}(\underline{n}) \subseteq \mathfrak{b}$ for all $\underline{n} \geq \underline{m}$. 
Proof. First of all, notice that $(\mathcal{O}: \overline{\mathcal{O}})=\{x \in \overline{\mathcal{O}} \mid \underline{v}(x) \geq \gamma\}$. Then $x \in z^{-1} \mathcal{O}$ for all $x \in \overline{\mathcal{O}}$ and $z \in \mathfrak{b}$, hence $(\mathcal{O}: \overline{\mathcal{O}}) \subseteq z^{-1} \mathfrak{b}$ for all $z \in \mathfrak{b}$ and $z \cdot(\mathcal{O}: \overline{\mathcal{O}})=\{x \mid \underline{v}(x) \geq \gamma+\underline{v}(z)\} \subseteq \mathfrak{b}$ for all $z \in \mathfrak{b}$. Therefore $J^{\mathcal{K}}(\underline{n}) \subset \mathfrak{b}$ for every $\underline{n} \geq \underline{v}(z)$.

(3.4) Lemma: We have:

(a) $\underline{0} \leq \gamma^{\mathfrak{b}} \leq \underline{v}((\mathcal{O}: \overline{\mathcal{O}}))=\gamma$.

(b) If $\mathfrak{b} \cdot \overline{\mathcal{O}}=\overline{\mathcal{O}}$, then $\gamma^{\mathfrak{b}}:=\min \left\{\underline{n} \mid \mathfrak{m}^{\underline{n}} \subseteq \mathfrak{b}\right\}$.

Proof. (a) From [13, Lemma 3.1], one has the inclusions

$$
(\mathcal{O}: \overline{\mathcal{O}}) \subseteq(\mathfrak{b}: \overline{\mathcal{O}}):(\mathfrak{b} \cdot \overline{\mathcal{O}}) \subseteq \overline{\mathcal{O}}
$$

and the statement follows. The assertion (b) can be deduced from the fact that $(\mathfrak{b}: \overline{\mathcal{O}}): \mathfrak{b}=(\mathfrak{b}: \overline{\mathcal{O}}):(\mathfrak{b} \cdot \overline{\mathcal{O}})$.

(3.5) In the rest of the section, the ring $\mathcal{O}$ will be assumed to be residually rational, i.e., $k=k_{i}$ for every $i \in\{1, \ldots, r\}$.

(3.6) For every $\underline{n}=\left(n_{1}, \ldots, n_{r}\right) \in \mathbb{Z}^{r}$ and for every $i \in\{1, \ldots, r\}$, define

$\Delta_{i}(\underline{n})=\left\{\left(\sigma_{1}, \ldots, \sigma_{r}\right) \in S(\mathfrak{b}) \mid \sigma_{i}=n_{i}\right.$ and $\sigma_{j}>\sigma_{i}$ for $\left.j \in\{1, \ldots, r\}, j \neq i\right\}$.

Moreover, for every $\underline{n} \in \mathbb{Z}^{r}$ define

$$
\Delta(\underline{n})=\bigcup_{i=1}^{r} \Delta_{i}(\underline{n}) .
$$

(3.7) Definition: The $S(\mathcal{O})$-module $S(\mathfrak{b})$ is said to be symmetric if there exists $\tau \in \mathbb{Z}^{r}$ such that, for every $\underline{v} \in \mathbb{Z}^{r}, \underline{v} \in S(\mathfrak{b})$ if and only if $\Delta(\tau-\underline{v})=\varnothing$.

(3.8) Proposition: Let $\mathcal{O}$ be residually rational. We have

(1) $\Delta\left(\gamma^{\mathfrak{b}}-\underline{v}-\underline{1}\right)=\varnothing$ for every $\underline{v} \in S(\mathfrak{b})$.

(2) If $S(\mathfrak{b})$ is symmetric then $\mathfrak{b}$ is self-dual.

(3) Suppose, in addition, that $k$ is an infinite field; if $\mathfrak{b}$ is a self-dual fractional ideal, then $S(\mathfrak{b})$ is symmetric.

Proof. (1) Let $\underline{v} \in S(\mathfrak{b})$. Then we have $J^{\mathfrak{b}}(\underline{v}) / J^{\mathfrak{b}}\left(\underline{v}+\underline{1}_{\{i\}}\right) \neq 0$ for every $i \in\{1, \ldots, r\}$, therefore $J^{\mathfrak{b}}\left(\gamma^{\mathfrak{b}}-\underline{v}-\underline{1}_{\{i\}}\right) / J^{\mathfrak{b}}\left(\gamma^{\mathfrak{b}}-\underline{v}\right) \neq 0$ for every $i \in\{1, \ldots, r\}$ and so $\Delta\left(\gamma^{\mathfrak{b}}-\underline{v}-\underline{1}\right)=\varnothing$. (2) Let $\underline{v}=\left(v_{1}, \ldots, v_{r}\right) \in \mathbb{Z}^{r}$ and take $i \in\{1, \ldots, r\}$. If $J^{\mathfrak{b}}(\underline{v}) / J^{\mathfrak{b}}\left(\underline{v}+\underline{1}_{\{i\}}\right) \neq 0$ then we must have that $J^{\mathfrak{b}}\left(\gamma^{\mathfrak{b}}-\underline{v}-\underline{1}_{\{i\}}\right) / J^{\mathfrak{b}}\left(\gamma^{\mathfrak{b}}-\underline{v}\right)=0$. Let us consider the case in which $J^{\mathfrak{b}}(\underline{v}) / J^{\mathfrak{b}}\left(\underline{v}+\underline{1}_{\{i\}}\right)=0$. There exists a vector $\underline{w}=\left(w_{1}, \ldots, w_{r}\right) \in \mathbb{Z}^{r}$ with $\Delta(\underline{w})=\varnothing, w_{i}=v_{i}$ and $w_{j}<v_{j}$ for every $j \in\{1, \ldots, r\}, j \neq i$. 
Since $S(\mathfrak{b})$ is symmetric, $\gamma^{\mathfrak{b}}-\underline{w}-\underline{1} \in S(\mathfrak{b})$. Now $\gamma_{j}^{\mathfrak{b}}-w_{j}-1 \geq$ $\gamma^{\mathfrak{b}}-v_{j}$ for every $j \in\{1, \ldots, r\}, j \neq i$, and $\gamma_{i}^{\mathfrak{b}}-w_{i}-1=\gamma_{i}^{\mathfrak{b}}-v_{i}-1$, hence for any regular element $z \in \mathcal{O}$ satisfying $\underline{v}(z)=\gamma^{\mathfrak{b}}-\underline{w}-\underline{1}$ it follows that $z \in J^{\mathfrak{b}}\left(\gamma^{\mathfrak{b}}-\underline{v}-\underline{1}_{\{i\}}\right), z \notin J^{\mathfrak{b}}\left(\gamma^{\mathfrak{b}}-\underline{v}\right)$, and therefore $J^{\mathfrak{b}}\left(\gamma^{\mathfrak{b}}-\underline{v}-\underline{1}_{\{i\}}\right) / J^{\mathfrak{b}}\left(\gamma^{\mathfrak{b}}-\underline{v}\right) \neq 0$. Theorem (2.19) implies that $\mathfrak{b}$ is self-dual. (3) Assume that $k$ is infinite. Let $\underline{v} \in \mathbb{Z}^{r}$ and assume that $\Delta\left(\gamma^{\mathfrak{b}}-\underline{v}-\underline{1}\right)=\varnothing$. Then $J^{\mathfrak{b}}\left(\gamma^{\mathfrak{b}}-\underline{v}-\underline{1}_{\{i\}}\right) / J^{\mathfrak{b}}\left(\gamma^{\mathfrak{b}}-\underline{v}\right)=0$ for every $i \in\{1, \ldots, r\}$, hence $J^{\mathfrak{b}}(\underline{v}) / J^{\mathfrak{b}}\left(\underline{v}+\underline{1}_{\{i\}}\right)$ for every $i \in\{1, \ldots, r\}$ by Theorem (2.17) and therefore $z \in S(\mathfrak{b})$ by Lemma (3.1). Thus $S(\mathfrak{b})$ is symmetric.

(3.9) Corollary: Let $k$ be an infinite field. The value semigroup $S(\mathcal{O})$ is symmetric if and only if the ring $\mathcal{O}$ is Gorenstein.

\section{Generalised Poincaré series of a Fractional ideal}

(4.1) Let $\mathfrak{b}$ be a fractional ideal in $\mathcal{O}$. The multi-index filtration $\left\{J^{\mathfrak{b}}(\underline{v})\right\}$ defines a Laurent series

$$
L\left(\mathfrak{b}, t_{1}, \ldots, t_{r}\right):=\sum_{\underline{v} \in \mathbb{Z}^{r}} \operatorname{dim}_{k}\left(J^{\mathfrak{b}}(\underline{v}) / J^{\mathfrak{b}}(\underline{v}+\underline{1})\right) \cdot \underline{t} \underline{\underline{v}} \in \mathbb{Z} \llbracket t_{1}, \ldots, t_{r} \rrbracket,
$$

where $\underline{t} \underline{v}:=t_{1}^{v_{1}} \cdot \ldots \cdot t_{r}^{v_{r}}$. We will write $L(\mathfrak{b}, \underline{t})$ instead of $L\left(\mathfrak{b}, t_{1}, \ldots, t_{r}\right)$ if the number of variables is clear from the context.

(4.2) There is a priori no fixed way to choose a suitable coefficient in $L(\mathfrak{b}, \underline{t})$. We may consider the following spaces:

(1) $J^{\mathfrak{b}}(\underline{v}) / J^{\mathfrak{b}}(\underline{v}+\underline{1})$;

(2) $J^{\mathfrak{b}}(\underline{v}) / J^{\mathfrak{b}}(\underline{v}+\underline{1}) \backslash \bigcup_{i=1}^{r} J^{\mathfrak{b}}\left(\underline{v}+\underline{1}_{\{i\}}\right) / J^{\mathfrak{b}}(\underline{v}+\underline{1})$;

(3) $J^{\mathfrak{b}}(\underline{v}) \backslash \bigcup_{i=1}^{r} J^{\mathfrak{b}}\left(\underline{v}+\underline{1}_{\{i\}}\right)$.

Filtration (1) is related to the semigroup of values of the ring, (2) defines the Poincaré series in terms of the extended semigroup of the ring, and (3) introduces the Poincaré series as an integral with respect to the Euler characteristic. Exactly this last point of view makes clear the association between the dimension of a vector space and the Euler characteristic $\chi$ of its projectivisation, namely:

$$
\operatorname{dim}_{k}(J(\underline{v}) / J(\underline{v}+\underline{1}))=\chi(\mathbb{P}(J(\underline{v}) / J(\underline{v}+\underline{1}))) .
$$

(4.3) We can also choose other measures than $\chi$, for instance the socalled generalised Euler characteristic $\chi_{g}$. It is a sort of motivic Euler characteristic which makes use of the notion of Grothendieck ring. The Grothendieck ring $K_{0}\left(\boldsymbol{V}_{k}\right)$ is defined to be the free Abelian group on 
isomorphism classes $[X]$ of quasi-projective schemes $X$ of finite type over $k$ subject to the following relations:

(1) $\left[X_{1}\right]=\left[X_{2}\right]$ if $X_{1} \cong X_{2}$ for $X_{1}, X_{2} \in \nu_{k}$;

(2) $[X]=[X \backslash Z]+[Z]$ for a closed subscheme $Z$ of $X \in \nu_{k}$;

and taking the fibred product as multiplication:

(3) $\left[X_{1}\right] \cdot\left[X_{2}\right]=\left[X_{1} \times_{k} X_{2}\right]$ for $X_{1}, X_{2} \in \nu_{k}$.

(4.4) Let $k[T]$ be the polynomial ring in one indeterminate $T$ over the field $k$. The affine scheme $\operatorname{Spec}(k[T])$ over $k$ is the affine line over $k$, which will be denoted by $\mathbb{A}_{k}^{1}$. The class of the affine line in $K_{0}\left(\boldsymbol{\nu}_{k}\right)$, denoted by $\mathbb{L}$, is called the Lefschetz class of $K_{0}\left(\boldsymbol{\nu}_{k}\right)$.

(4.5) Let $p$ be a non-negative integer and let $J_{\mathcal{O}}^{p}$ be the space of $p$ jets over $\mathcal{O}$, which is a finite-dimensional $k$-vector space of dimension $d(p)$. Let us consider its projectivisation $\mathbb{P} J_{\mathcal{O}}^{p}$ and let us adjoin one point to this (that is, $\mathbb{P}^{*} J_{\mathcal{O}}^{p}=\mathbb{P} J_{\mathcal{O}}^{p} \cup\{*\}$ with $*$ representing the added point) in order to have a well-defined map $\pi_{p}: \mathbb{P O} \rightarrow \mathbb{P}^{*} J_{\mathcal{O}}^{p}$. A subset $X \subset \mathbb{P O}$ is said to be cylindric if there exists a constructible subset $Y \subset \mathbb{P} J_{\mathcal{O}}^{p} \subset \mathbb{P}^{*} J_{\mathcal{O}}^{p}$ such that $X=\pi_{p}^{-1}(Y)$.

(4.6) The generalised Euler characteristic $\chi_{g}(X)$ of a cylindric subset $X$ is the element $[Y] \cdot \mathbb{L}^{-d(p)}$ in the ring $K_{0}\left(\nu_{k}\right)_{(\mathbb{L})}$, where $Y=\pi_{p}^{-1}(X)$ is a constructible subset of $\mathbb{P} \mathcal{O}$. Note that $\chi_{g}(X)$ is well-defined, because if $X=\pi_{q}^{-1}\left(Y^{\prime}\right), Y^{\prime} \subset \mathbb{P} J_{\mathcal{O}}^{q}$ and $p \geq q$, then $Y$ is a locally trivial fibration over $Y^{\prime}$ and therefore $[Y]=\left[Y^{\prime}\right] \cdot \mathbb{L}^{d(p)-d(q)}$.

(4.7) As in [7], we can extend these definitions to subsets of $\mathcal{K}$ (in particular to fractional ideals): a subset $X \subseteq \mathcal{K}$ is called cylindric if there exists a non-zero divisor element $z \in \mathcal{O}$ such that the set $z X$ is a subset of $\mathcal{O}$ and is cylindric. In this situation, the generalised Euler characteristic is

$$
\chi_{g}(X):=\frac{\chi_{g}(z X)}{\chi_{g}(z \mathcal{O})}
$$

Let $\mathfrak{a} \subseteq \mathcal{O}$ be an ideal of $\mathcal{O}$. Since $\mathfrak{a}$ is $\mathfrak{m}$-primary, we have $\mathfrak{m}^{p+1} \subseteq \mathfrak{a}$. Let $\overline{\mathfrak{a}}$ be the ideal $\mathfrak{a} / \mathfrak{m}^{p+1}$ of $\mathcal{O} / \mathfrak{m}^{p+1}$ so that $\pi_{p}^{-1}(\overline{\mathfrak{a}})=\mathfrak{a}$. As $\mathcal{O} / \mathfrak{m}^{p+1}$ is a finite-dimensional $k$-vector space, the ideal $\overline{\mathfrak{a}}$ is constructible. Then $\mathfrak{a}$ is cylindric and we get

$$
\begin{aligned}
\chi_{g}(\mathfrak{a}) & =[\overline{\mathfrak{a}}] \cdot \mathbb{L}^{-d(p)} \\
& =\mathbb{L}^{\operatorname{dim}_{k}\left(\mathfrak{a} / \mathfrak{m}^{p+1}\right)-d(p)} \\
& =\mathbb{L}^{\operatorname{deg}(\mathfrak{a})} .
\end{aligned}
$$


In particular, $\chi_{g}\left(\mathfrak{m}^{p+1}\right)=\mathbb{L}^{-d(p)}$.

(4.8) Let $G$ be an abelian group with countable many values. Let $X$ be a cylindric subset of $\mathcal{K}$. A function $\psi: X \rightarrow G$ is called cylindric if the set $\psi^{-1}(a) \subseteq \mathcal{K}$ is cylindric for all $a \in G \backslash\{0\}$. The integral of $\psi$ over $X$ with respect to the generalised Euler characteristic is

$$
\int_{X} \psi d \chi_{g}:=\sum_{a \in G \backslash\{0\}} \chi_{g}\left(\psi^{-1}(a)\right) \cdot a,
$$

if this sum makes sense in $K_{0}\left(\boldsymbol{\nu}_{k}\right)_{(\mathbb{L})} \otimes_{\mathbb{Z}} G$; in such a case, the function $\psi$ is said to be integrable.

(4.9) Remark: Let $\psi: \mathbb{P} X \rightarrow G$ be a cylindric function of $X$. Let us denote by $\psi^{\prime}: X \rightarrow G$ the function induced by $\psi$ on $X$ with $\psi^{\prime}(0)=0$. Then the function $\psi^{\prime}$ is cylindric if and only if $\psi$ is cylindric; in this case we have

$$
(\mathbb{L}-1) \int_{\mathbb{P} X} \psi d \chi_{g}=\int_{X} \psi^{\prime} d \chi_{g}
$$

(cf. [7, (2.7)]).

We define now the generalised Poincaré series of the projectivisation of the fractional ideal $\mathfrak{b}$ :

(4.10) Definition: The generalised Poincaré series of a multi-index filtration given by the ideals $J(\underline{v})$ is the integral

$$
P_{g}(\mathfrak{b}, \underline{t}, \mathbb{L}):=\int_{\mathbb{P} \mathfrak{b}} \underline{t}^{\underline{v}(z)} d \chi_{g} \in K_{0}\left(\boldsymbol{\nu}_{k}\right)_{(\mathbb{L})} \llbracket t_{1}, \ldots, t_{r} \rrbracket,
$$

where $\underline{t}^{\underline{v}(z)}:=t_{1}^{v_{1}(z)} \cdot \ldots \cdot t_{r}^{v_{r}(z)}$ is considered as a (cylindric) function on $\mathbb{P O}$ with values in $\mathbb{Z} \llbracket t_{1}, \ldots, t_{r} \rrbracket$ (the vector $\underline{v}(z)$ is supposed to be $\underline{0}$ as soon as $v_{i}(z)=\infty$ for at least one $\left.i \in\{1, \ldots, r\}\right)$.

(4.11) Remark: Notice that if $\mathfrak{b}=\mathcal{O}$, then $P_{g}(\mathcal{O}, \underline{t}, \mathbb{L})$ is the generalised Poincaré series of a filtration $J(\underline{v})$ over the projectivization of the ring $\mathcal{O}$ introduced in [4, Section 2, p. 198] for the case of the ring $\mathcal{O}_{V, 0}$ of functions on a germ $(V, 0)$ of a complex analytic variety.

(4.12) Let us define the degree of a fractional $\mathcal{O}$-ideal by the following two properties: (i) $\operatorname{deg}(\mathcal{O}):=0$; (ii) for every two fractional $\mathcal{O}$-ideals $\mathfrak{a}, \mathfrak{b}$, one has $\operatorname{deg}(\mathfrak{a})-\operatorname{deg}(\mathfrak{b})=\operatorname{dim}(\mathfrak{a} / \mathfrak{b})$ whenever $\mathfrak{a} \supseteq \mathfrak{b}$. Now, if we define

$$
L_{g}(\mathfrak{b}, \underline{t}, \mathbb{L}):=\sum_{\underline{v} \in \mathbb{Z}^{r}}\left(\mathbb{L}^{\operatorname{deg}\left(J^{\mathfrak{b}}(\underline{v})\right)}-\mathbb{L}^{\operatorname{deg}\left(J^{\mathfrak{b}}(\underline{v}+\underline{1})\right)}\right) \cdot \underline{t}^{\underline{v}},
$$

then we get 


\section{(4.13) Lemma:}

$$
P_{g}(\mathfrak{b}, \underline{t}, \mathbb{L})=\frac{\prod_{i=1}^{r}\left(t_{i}-1\right) L_{g}(\mathcal{O}, \mathfrak{b} ; \underline{t})}{t_{1} \cdot \ldots \cdot t_{r}-1}
$$

Proof. The result may be proved in much the same way as in [4, Proposition 2].

We describe now the functional equations for the series $P_{g}(\mathfrak{b}, \underline{t}, \mathbb{L})$. First of all, we state the following two results, due to Stöhr (see [12] and [13]). We include the proofs by the sack of completeness.

(4.14) Lemma: Let $\mathfrak{a}, \mathfrak{b}$ be fractional ideals of $\mathcal{O}$ such that $\mathfrak{a} \supseteq \mathfrak{b}$. We have

$$
\lambda\left(\mathfrak{b}^{*} \cap \mathfrak{a} / \mathfrak{b} \cap \mathfrak{a}^{*}\right)=\lambda(\mathfrak{a} / \mathfrak{b}) .
$$

Proof. By definition of the ideal $\mathfrak{c}$ we have $\mathfrak{c}: \mathfrak{b}^{*}=\mathfrak{b}$, hence $\mathfrak{b}^{*} \cap \mathfrak{a}=$ $(\mathfrak{c}: \mathfrak{b}) \cap\left(\mathfrak{c}: \mathfrak{a}^{*}\right)=\mathfrak{c}:\left(\mathfrak{b}+\mathfrak{a}^{*}\right)$ and $\operatorname{deg}\left(\mathfrak{b}^{*} \cap \mathfrak{a}\right) \stackrel{\star}{=} \operatorname{deg}(\mathfrak{c})+\operatorname{deg}\left(\mathfrak{b}+\mathfrak{a}^{*}\right)$. From the isomorphisms

$$
\mathfrak{b}+\mathfrak{a}^{*} / \mathcal{O} \cong \mathfrak{b}+\mathfrak{a}^{*} / \mathfrak{a}^{*}+\mathfrak{a}^{*} / \mathcal{O} \cong \mathfrak{b} / \mathfrak{b} \cap \mathfrak{a}^{*}+\mathfrak{a}^{*} / \mathcal{O}
$$

it follows that $\operatorname{deg}\left(\mathfrak{b}+\mathfrak{a}^{*}\right)=\operatorname{deg}(\mathfrak{b})-\operatorname{deg}\left(\mathfrak{b} \cap \mathfrak{a}^{*}\right)+\operatorname{deg}(\mathfrak{c})-\operatorname{deg}(\mathfrak{a})$, and by $\star$ we get

$$
\operatorname{deg}\left(\mathfrak{b}^{*} \cap \mathfrak{a}\right)=\operatorname{deg}\left(\mathfrak{b} \cap \mathfrak{a}^{*}\right)+\operatorname{deg}(\mathfrak{a})-\operatorname{deg}(\mathfrak{b}),
$$

which proves the statement.

(4.15) Lemma: Let $\mathfrak{a}$ be a fractional ideal of $\overline{\mathcal{O}}$. The following assertions hold:

(a) Let $t_{i}$ be a generator of the ideal $\mathfrak{m}_{i}$ for every $i \in\{1, \ldots, r\}$. The fractional ideals $\mathfrak{a}$ of $\mathcal{O}$ are of the form $\underline{t}^{-\underline{n}} \cdot \mathfrak{b}$, where $\underline{\underline{n}}:=$ $t_{1}^{n_{1}} \cdot \ldots \cdot t_{r}^{n_{r}}$ for some $\underline{n}=\left(n_{1}, \ldots, n_{r}\right) \in \mathbb{Z}^{r}$ and being $\mathfrak{b}$ a fractional ideal of $\mathcal{O}$ such that $\mathfrak{b} \cdot \overline{\mathcal{O}}=\overline{\mathcal{O}}$.

(b) There exists some $\underline{v} \in \mathbb{Z}^{r}$ such that $\mathfrak{a}=J^{\mathcal{K}}(\underline{v})$ and $\mathfrak{a}^{*}=$ $J^{\mathcal{K}}(-\underline{v})$.

(c) For some $\underline{v} \in \mathbb{Z}^{r}$, we have $\operatorname{deg}(\mathfrak{a})=\delta-\underline{v} \cdot \underline{d}$.

Proof. (a) Since $\mathfrak{a}$ is a fractional ideal of $\mathcal{O}, \mathfrak{a} \overline{\mathcal{O}}$ is a fractional ideal of $\overline{\mathcal{O}}$. By Remark 2, we have that $\mathfrak{a} \overline{\mathcal{O}}$ must be of the form $\mathfrak{m}_{1}^{n_{1}} \cdot \ldots \cdot \mathfrak{m}_{r}^{n_{r}}$ for some $\underline{n} \in \mathbb{Z}^{r}$. That is,

$$
\mathfrak{a} \overline{\mathcal{O}}=t_{1}^{n_{1}} \overline{\mathcal{O}} \cdot \ldots \cdot t_{r}^{n_{r}} \overline{\mathcal{O}}=\underline{t}^{\underline{n}} \cdot \overline{\mathcal{O}}
$$

i.e., $\underline{t}^{-\underline{n}} \cdot \mathfrak{a} \cdot \overline{\mathcal{O}}=\overline{\mathcal{O}}$ for some $\underline{n} \in \mathbb{Z}^{r}$. Then, it suffices to take $\mathfrak{b}=\underline{t}^{-\underline{n}} \cdot \mathfrak{a}$ and the claim follows. 
(b) Let $\mathfrak{c}$ be a canonical ideal of $\mathcal{O}$. We know that

$$
\begin{aligned}
\operatorname{deg}(\mathfrak{c}) & =\operatorname{deg}(\mathfrak{c}: \mathcal{O}) \\
& =\operatorname{deg}(\mathfrak{c}: \overline{\mathcal{O}})+\operatorname{dim}(\overline{\mathcal{O}} / \mathcal{O}) \\
& =\operatorname{deg}(\mathfrak{c}: \overline{\mathcal{O}})+\delta .
\end{aligned}
$$

If we multiply the ideal $\mathfrak{c}$ by a convenient element of $\mathcal{K}$, then we may assume $\mathfrak{c}: \overline{\mathcal{O}}=\overline{\mathcal{O}}$. The ideal $\mathfrak{c}: \overline{\mathcal{O}}$ is fractional, hence by (a) there is some $\underline{v} \in \mathbb{Z}^{r}$ such that $\mathfrak{c}: \overline{\mathcal{O}}=\underline{t^{v}} \cdot \overline{\mathcal{O}}$, which is equivalent to $\overline{\mathcal{O}}=$ $\left(\underline{t}^{-\underline{v}} \cdot \mathfrak{c}\right): \overline{\mathcal{O}}$. Then we have

$$
\mathfrak{a}^{*} \mathfrak{c}: \mathfrak{a}=\mathfrak{c}: J^{\mathcal{K}}(\underline{v})=\mathfrak{c}:\left(\underline{t}^{\underline{v}} \cdot \overline{\mathcal{O}}\right)=\underline{t}^{-\underline{v}}(\mathfrak{c}: \overline{\mathcal{O}})=\underline{t}^{-\underline{v}} \cdot \overline{\mathcal{O}}=J^{\mathcal{K}}(-\underline{v}) .
$$

(c) By $(a)$, there is some $\underline{v} \in \mathbb{Z}^{r}$ such that $\mathfrak{a}=J^{\mathcal{K}}(\underline{v})$. Since $d_{i}=$ $\operatorname{dim}\left(\overline{\mathcal{O}} / \mathfrak{m}_{i}\right)$ for all $1 \leq i \leq r$, by the Chinese Remainder Theorem we have

$$
\begin{aligned}
\operatorname{dim}\left(\overline{\mathcal{O}} / J^{\mathcal{K}}(\underline{v})\right) & =\operatorname{deg}(\overline{\mathcal{O}})-\operatorname{deg}\left(J^{\mathcal{K}}(\underline{v})\right) \\
& =\sum_{i=1}^{r} d_{i} \cdot v_{i} \\
& =\underline{d} \cdot \underline{v} .
\end{aligned}
$$

Since $\delta=\operatorname{dim}(\overline{\mathcal{O}} / \mathcal{O})=\operatorname{deg}(\overline{\mathcal{O}})$, we have

$$
\begin{aligned}
\operatorname{deg}\left(J^{\mathcal{K}}(\underline{v})\right) & =\operatorname{deg}(\overline{\mathcal{O}})-\underline{d} \cdot \underline{v} \\
& =\delta-\underline{d} \cdot \underline{v} .
\end{aligned}
$$

Next proposition relates the degree of the ideal $J^{\mathfrak{b}^{*}}(\underline{v})$ and the value $\gamma^{\mathfrak{b}}$.

(4.16) Proposition: For every $\underline{v} \in \mathbb{Z}^{r}$, we have

$$
\begin{aligned}
\operatorname{deg}\left(J^{\mathfrak{b}^{*}}(\underline{v})\right) & =\operatorname{deg}\left(J^{\mathfrak{b}}\left(\gamma^{\mathfrak{b}}-\underline{v}\right)\right)+\operatorname{dim}(\mathfrak{b} /(\mathfrak{b}: \overline{\mathcal{O}}))-\underline{v} \cdot \underline{d} \\
& =\operatorname{deg}\left(J^{\mathfrak{b}}\left(\gamma^{\mathfrak{b}}-\underline{v}\right)\right)+\operatorname{dim}\left(\mathfrak{b}^{*} \cdot \overline{\mathcal{O}} / \mathfrak{b}^{*}\right)-\underline{v} \cdot \underline{d}
\end{aligned}
$$

where $\underline{v} \cdot \underline{d}:=v_{1} d_{1}+\ldots+v_{r} d_{r}$.

Proof. The second equality holds by Lemma (2.11). Moreover, since $J^{\mathfrak{b}}(\underline{v})=\mathfrak{b} \cap J^{\mathcal{K}}(\underline{v})$ for $\underline{v} \in \mathbb{Z}^{r}$, by Lemma (4.14) and Lemma (4.15) it follows that

$$
\operatorname{deg}\left(\mathfrak{b}^{*} \cap J^{\mathcal{K}}(\underline{v})\right)=\operatorname{dim}(\mathfrak{b} / \mathfrak{b}: \overline{\mathcal{O}})+\operatorname{deg}\left(J^{\mathcal{K}}(-\underline{v}) \cdot \overline{\mathcal{O}^{*}} \cap \mathfrak{b}\right)-\underline{v} \cdot \underline{d} .
$$

The definition of $\gamma^{\mathfrak{b}}$ allows us now to conclude. 
As a consequence we obtained the following result due to Moyano and Zúñiga ([11, Lemma 9])

(4.17) Corollary: The ring $\mathcal{O}$ is Gorenstein if and only if

$$
\ell(\gamma-\underline{v})-\ell(\underline{v})=\delta-\underline{v} \cdot \underline{d}
$$

for every $\underline{v} \in \mathbb{Z}^{r}$.

Proof. It is just to apply Proposition (4.16) to $\mathfrak{b}=\mathcal{O}$. Notice that we have $\mathcal{O}=\mathcal{O}^{*}$ (cf. (2.7)) because $\mathcal{O}$ is Gorenstein.

(4.18) Remark: Notice that $\delta-d=\ell(\gamma-\underline{1})-\rho$, if the ring is Gorenstein. It follows from Corollary (4.17), because $\delta-\underline{d} \cdot \underline{1}=\ell(\gamma-$ $\underline{1})-\ell(\underline{1})$ and $\ell(\underline{1})=\rho$.

Proposition (4.16) allows us to describe the functional equations for the generalised Poincaré series:

(4.19) Theorem:

$$
L_{g}\left(\mathfrak{b}, \mathbb{L}^{d_{1}} t_{1}, \ldots, \mathbb{L}^{d_{r}} t_{r}, \mathbb{L}\right)=\mathbb{L}^{\operatorname{dim}\left(\mathfrak{b}^{*} \cdot \overline{\mathcal{O}} / \mathfrak{b}^{*}\right)-d} \cdot \underline{t}^{\gamma^{\mathfrak{b}}-\underline{1}} \cdot L_{g}\left(\mathfrak{b}^{*} ; \underline{t}^{-1}, \mathbb{L}\right) .
$$

Proof. Let $A(\mathfrak{b}, \underline{t}):=\sum_{\underline{v} \in \mathbb{Z}^{r}} \mathbb{L}^{\operatorname{deg}\left(J^{\mathfrak{b}}(\underline{v})\right)} \cdot \underline{t}^{\underline{v}}$. Then

$$
\begin{aligned}
L_{g}(\mathfrak{b}, \underline{t}, \mathbb{L}) & =\sum_{\underline{v} \in \mathbb{Z}^{r}}\left(\mathbb{L}^{\operatorname{deg}\left(J^{\mathfrak{b}}(\underline{v})\right)}-\mathbb{L}^{\operatorname{deg}\left(J^{\mathfrak{b}}(\underline{v}+\underline{1})\right)}\right) \cdot \underline{t}^{\underline{v}} \\
& =\left(1-\underline{t}^{-1}\right) \cdot A(\mathfrak{b}, \underline{t}) .
\end{aligned}
$$

We apply now Proposition (4.16) to obtain

$$
A\left(\mathfrak{b}, \mathbb{L}^{d_{1}} t_{1}, \ldots, \mathbb{L}^{d_{r}} t_{r}\right)=\mathbb{L}^{\operatorname{dim}\left(\mathfrak{b}^{*} \cdot \overline{\mathcal{O}} / \mathfrak{b}^{*}\right)} \cdot \underline{\gamma}^{\gamma^{\mathfrak{b}}} \cdot A\left(\mathfrak{b}^{*}, t_{1}^{-1}, \ldots, t_{r}^{-1}\right) .
$$

Moreover, taking the inverse of $\underline{t}$, we have

$$
L_{g}\left(\mathfrak{b}^{*}, \underline{t}^{-1}, \mathbb{L}\right)=(1-\underline{t}) \cdot A\left(\mathfrak{b}^{*}, \underline{t}^{-1}\right) .
$$


Therefore

$$
\begin{aligned}
L_{g}\left(\mathcal{O}, \mathfrak{b}, \mathbb{L}^{d_{1}} t_{1}, \ldots, \mathbb{L}^{d_{r}} t_{r}\right) & =\frac{\mathbb{L}^{d} \cdot \underline{t}-1}{\mathbb{L}^{d} \cdot \underline{t}} \cdot A\left(\mathfrak{b}, \mathbb{L}^{d_{1}} t_{1}, \ldots, \mathbb{L}^{d_{r}} t_{r}\right) \\
& =\frac{\mathbb{L}^{d} \cdot \underline{t}-1}{\mathbb{L}^{d} \cdot \underline{t}} \cdot \mathbb{L}^{\operatorname{dim}\left(\mathfrak{b}^{*} \cdot \overline{\mathcal{O}} / \mathfrak{b}^{*}\right)} \cdot \underline{t}^{\gamma^{\mathfrak{b}}} \cdot A\left(\mathfrak{b}^{*}, t_{1}^{-1}, \ldots, t_{r}^{-1}\right) \\
& =\mathbb{L}^{d} \cdot \underline{t}-1 \\
(\dagger) & \frac{1}{1-t} \cdot \mathbb{L}^{\operatorname{dim}\left(\mathfrak{b}^{*} \cdot \overline{\mathcal{O}} / \mathfrak{b}^{*}\right)} \cdot \underline{t}^{\gamma^{\mathfrak{b}}} \cdot L_{g}\left(\mathfrak{b}^{*}, \underline{t}^{-1}, \mathbb{L}\right) \\
& =\frac{\mathbb{L}^{d} \cdot \underline{t}-1}{1-\underline{t}} \cdot \mathbb{L}^{\operatorname{dim}\left(\mathfrak{b}^{*} \cdot \overline{\mathcal{O}} / \mathfrak{b}^{*}\right)-d} \cdot \underline{t}^{\gamma^{\mathfrak{b}}-\underline{1}} \cdot L_{g}\left(\mathfrak{b}^{*}, \underline{t}^{-1}, \mathbb{L}\right) .
\end{aligned}
$$

\section{(4.20) Corollary:}

$P_{g}\left(\mathfrak{b}, \mathbb{L}^{d_{1}} t_{1}, \ldots, \mathbb{L}^{d_{r}} t_{r}, \mathbb{L}\right)=\mathbb{L}^{\operatorname{dim}\left(\mathfrak{b}^{*} \cdot \overline{\mathcal{O}} / \mathfrak{b}^{*}\right)-d} \cdot{\underline{\gamma^{\mathfrak{b}}}}^{-\underline{1}} \cdot \frac{\prod_{i=1}^{r}\left(1-\mathbb{L}^{d_{i}} t_{i}\right)}{\prod_{i=1}^{r}\left(t_{i}-1\right)} \cdot P_{g}\left(\mathfrak{b}^{*}, \underline{t}^{-1}, \mathbb{L}\right)$.

(4.21) Corollary: If $\mathfrak{b}=\mathcal{O}$, then we have

$P_{g}\left(\mathcal{O}, \mathbb{L}^{d_{1}} t_{1}, \ldots, \mathbb{L}^{d_{r}} t_{r}, \mathbb{L}\right)=\mathbb{L}^{\operatorname{dim}\left(\mathcal{O}^{*} \cdot \overline{\mathcal{O}} / \mathcal{O}^{*}\right)-d} \cdot \underline{t}^{\gamma-1} \cdot \frac{\prod_{i=1}^{r}\left(1-\mathbb{L}^{d_{i}} t_{i}\right)}{\prod_{i=1}^{r}\left(t_{i}-1\right)} \cdot P_{g}\left(\mathcal{O}^{*}, \underline{t}^{-1}, \mathbb{L}\right)$.

Furthermore, if $\mathcal{O}$ is Gorenstein, then we obtain

$P_{g}\left(\mathcal{O}, \mathbb{L}^{d_{1}} t_{1}, \ldots, \mathbb{L}^{d_{r}} t_{r}, \mathbb{L}\right)=\mathbb{L}^{\delta-d} \cdot \underline{t}^{\gamma-\underline{1}} \cdot \frac{\prod_{i=1}^{r}\left(1-\mathbb{L}^{d_{i}} t_{i}\right)}{\prod_{i=1}^{r}\left(t_{i}-1\right)} \cdot P_{g}\left(\mathcal{O}, \underline{t}^{-1}, \mathbb{L}\right)$.

Proof. It is a straight consequence of Corollary (4.20) and Corollary (4.17).

\section{Extended Generalised Value ideal Poincaré SeRies}

Let $\mathcal{O}$ be a one-dimensional Cohen-Macaulay local Noetherian ring having a perfect coefficient field $K$. Campillo, Delgado and GuseinZade introduced in [1] the notion of extended semigroup of a germ of complex plane curve singularity. We want now to define the concept of extended value ideal of a fractional ideal $\mathfrak{b}$. Let us preserve notations as in (2.1). Remember that the ideal $\mathfrak{m}_{j} V_{j}$ is regular maximal of $V_{j}$ and $V_{j}=\overline{\mathcal{O}}_{\mathfrak{m}_{j}}$ for every $j \in\{1, \ldots, r\}$.

If $K_{j}$ is a coefficient field of $V_{j}$ and $t_{j}$ is an indeterminate over $K_{j}$, then one can identify $V_{j} \cong K_{j} \llbracket t_{j} \rrbracket$ and $v_{j}$ with the order function respect to 
$t_{j}$ in $K_{j} \llbracket t_{j} \rrbracket$ for every $j \in\{1, \ldots, r\}$. Thus

$$
\mathcal{O} \subset K_{1} \llbracket t_{1} \rrbracket \cap \ldots \cap K_{r} \llbracket t_{r} \rrbracket=\overline{\mathcal{O}} .
$$

Since $V_{j}$ is an $\mathcal{O}$-module of finite type, the field extensions $\mathcal{O} / \mathfrak{m} \hookrightarrow$ $\overline{\mathcal{O}} / \mathfrak{m}_{j}$ are finite for every $j \in\{1, \ldots r\}$. Furthermore, as $\mathcal{O} / \mathfrak{m}$ is assumed to be perfect, every such a extension is separable and therefore, for every coefficient field $K$ of $\mathcal{O}$ there exists a unique coefficient field $K_{j}$ of $V_{j}$ with $K \subset K_{j}$ which is isomorphic to $\overline{\mathcal{O}} / \mathfrak{m}_{j}$ for every $j \in\{1, \ldots, r\}$.

Let us consider the vector spaces $C^{\mathfrak{b}}(\underline{v}, i)=J^{\mathfrak{b}}(\underline{v}) / J^{\mathfrak{b}}\left(\underline{v}+\underline{1}_{\{i\}}\right)$ for every $i \in\{1, \ldots, r\}$ and the map:

$$
\begin{aligned}
j_{\underline{v}}: J^{\mathfrak{b}}(\underline{v}) & \longrightarrow C^{\mathfrak{b}}(\underline{v}, 1) \times \ldots \times C^{\mathfrak{b}}(\underline{v}, r) \\
z & \mapsto\left(j_{1}(z), \ldots, j_{r}(z)\right)=: \bar{j}_{\underline{v}}(z) .
\end{aligned}
$$

We can identify $\operatorname{Im} j_{\underline{v}} \cong C^{\mathfrak{b}}(\underline{v})=J^{\mathfrak{b}}(\underline{v}) / J^{\mathfrak{b}}(\underline{v}+\underline{1})$ and define the set

$$
F_{\underline{v}}^{\mathfrak{b}}:=C^{\mathfrak{b}}(\underline{v}) \cap\left(\left(C^{\mathfrak{b}}(\underline{v}, 1) \backslash\{0\}\right) \times \ldots \times\left(C^{\mathfrak{b}}(\underline{v}, r) \backslash\{0\}\right)\right) .
$$

\section{(5.1) Lemma:}

$$
F_{\underline{v}}^{\mathfrak{b}}=C^{\mathfrak{b}}(\underline{v}) \cap\left(K_{1}^{*} \times \ldots \times K_{r}^{*}\right) .
$$

Proof. It is enough to define an isomorphism $\varphi_{\underline{v}}: C^{\mathfrak{b}}(\underline{v}, 1) \backslash\{0\} \rightarrow K_{1}^{*}$. Let $z \in \mathfrak{b} \backslash\{0\}$ with $v_{1}(z)=v_{1}$. We have that $z=a_{1}(z) t_{1}^{v_{1}(z)}$ with $a_{1}(z) \in K_{1}^{*}$, thus $\varphi_{\underline{v}}$ can be defined by $z \mapsto a_{1}(z)$.

(5.2) For every $\underline{v} \in \mathbb{Z}^{r}$ we write

$$
F_{\underline{v}}^{\mathfrak{b}}=\left(J^{\mathfrak{b}}(\underline{v}) / J^{\mathfrak{b}}(\underline{v}+\underline{1})\right) \backslash \bigcup_{i=1}^{r}\left(J^{\mathfrak{b}}\left(\underline{v}+\underline{1}_{\{i\}}\right) / J^{\mathfrak{b}}(\underline{v}+\underline{1})\right),
$$

i.e., $F_{\underline{v}}^{\mathfrak{b}}$ is the complement to an arrangement of vector subspaces in a vector space (it is not a vector subspace itself). Notice that this is precisely the space (2) in (4.2).

(5.3) Definition: For every fractional ideal $\mathfrak{b}$ of $\mathcal{O}$ we define the set $\widehat{S}(\mathfrak{b})$ to be the union of the subspaces $F_{\underline{v}}^{\mathfrak{b}}$ for all $\underline{v} \in \mathbb{Z}^{r}$. The spaces $F_{\underline{v}}^{\mathfrak{b}}$ are called fibres of $\widehat{S}(\mathfrak{b})$.

Notice that, if $\mathfrak{b}=\mathcal{O}$, then $\widehat{S}(\mathcal{O})$ is the extended semigroup of the ring $\mathcal{O}$ (see [1] for further details).

(5.4) The group $K^{*}$ of non-zero elements of $K$ acts freely on $\mathbb{Z}^{r} \times\left(K_{1}^{*} \times\right.$ $\ldots \times K_{r}^{*}$ ) (by multiplication of all coordinates in $K_{1}^{*} \times \ldots \times K_{r}^{*}$ ). The 
corresponding factor space $\mathbb{Z}^{r} \times\left(K_{1}^{*} \times \ldots \times K_{r}^{*}\right) / K^{*}=\mathbb{Z}^{r} \times \mathbb{P}\left(K_{1}^{*} \times\right.$ $\left.\ldots \times K_{r}^{*}\right)=\sum_{\underline{v} \in \mathbb{Z}^{r}} \mathbb{P}\left(K_{1}^{*} \times \ldots \times K_{r}^{*}\right) \underline{t} \underline{\underline{v}}$ has the natural structure of semigroup. The set $\widehat{S}(\mathfrak{b}) \subset \mathbb{Z}^{r} \times\left(K_{1}^{*} \times \ldots \times K_{r}^{*}\right)$ is invariant with respect to the $K^{*}$-action. The factor space

$$
\mathbb{P} \widehat{S}(\mathfrak{b})=\widehat{S}(\mathfrak{b}) / K^{*}
$$

is called the projectivisation of $\widehat{S}(\mathfrak{b})$ (it is also a graded $S(\mathcal{O})$-module in a natural sense).

From the previous definitions, the projectivisation of $\widehat{S}(\mathfrak{b})$ can be described as

$$
\mathbb{P} \widehat{S}(\mathfrak{b})=\sum_{\underline{v} \in \mathbb{Z}^{r}} \mathbb{P} F_{\underline{v}}^{\mathfrak{b}} \cdot \underline{t^{\underline{v}}},
$$

where $\mathbb{P} F_{\underline{v}}^{\mathfrak{b}}=F_{\underline{v}}^{\mathfrak{b}} / K^{*}$ is the projectivisation of the fibre $F_{\underline{v}}^{\mathfrak{b}}$. For $\underline{v} \in$ $\widehat{S}(\mathfrak{b})$, the space $\bar{P} F_{\underline{v}}^{\mathfrak{b}}$ is the complement to an arrangement of projective hyperplanes in a $\left(\overline{\operatorname{dim}}_{K}\left(J^{\mathfrak{b}}(\underline{v}) / J^{\mathfrak{b}}(\underline{v}+\underline{1})\right)-1\right)$-dimensional projective space $\mathbb{P}\left(J^{\mathfrak{b}}(\underline{v}) / J^{\mathfrak{b}}(\underline{v}+\underline{1})\right)$.

Set the Laurent series

$$
\chi_{g}(\mathbb{P} \widehat{S}(\mathfrak{b})):=\sum_{\underline{v} \in \mathbb{Z}^{r}} \chi_{g}\left(\mathbb{P} F_{\underline{v}}^{\mathfrak{b}}\right) \cdot \underline{t} \underline{\underline{v}} .
$$

On the other hand, we also have the extended generalised value ideal Poincaré series of a filtration $\left\{J^{\mathfrak{b}}(\underline{v})\right\}$ defined by $\underline{v}(z)=\left(v_{1}(z), \ldots, v_{r}(z)\right)$, for $z \in \mathfrak{b}$ :

$$
\widehat{P}_{g}\left(\mathfrak{b}, \underline{t}, \mathbb{L},\left\{v_{i}\right\}\right):=\int_{\mathbb{P} \widehat{S}(\mathfrak{b})} \underline{t}^{\underline{v}(z)} d \chi_{g}
$$

(we will use $\widehat{P}_{g}(\mathfrak{b}, \underline{t}, \mathbb{L})$ instead of $\widehat{P}_{g}\left(\mathfrak{b}, \underline{t}, \mathbb{L},\left\{v_{i}\right\}\right)$ when the filtration is clear from the context). Notice that if $\mathfrak{b}=\mathcal{O}$, then $\widehat{P}_{g}(\mathcal{O}, \underline{t}, \mathbb{L})$ coincides with the generalised semigroup Poincaré series defined in [4, p. 507].

All projectivisations $\mathbb{P} F_{\underline{v}}^{\mathfrak{b}}$ of the fibres $F_{\underline{v}}^{\mathfrak{b}}$ (i.e., all connected components of $\mathbb{P} \widehat{S}(\mathfrak{b}))$ are complements to arrangements of projective subspaces in finite dimensional projective spaces. We define

$$
\widehat{L}_{g}(\mathfrak{b}, \underline{t}, \mathbb{L}):=\sum_{\underline{v} \in \mathbb{Z}^{r}}\left[\mathbb{P}\left(J^{\mathfrak{b}}(\underline{v}) / J^{\mathfrak{b}}(\underline{v}+\underline{1})\right)\right] \cdot \underline{t}^{\underline{v}} .
$$

\section{(5.5) Proposition:}

$$
\chi_{g}(\mathbb{P} \widehat{S}(\mathfrak{b}))=\widehat{P}_{g}(\mathfrak{b}, \underline{t}, \mathbb{L})=\frac{\widehat{L}_{g}(\mathfrak{b}, \underline{t}, \mathbb{L}) \cdot \prod_{i=1}^{r}\left(t_{i}-1\right)}{t_{1} \cdot \ldots \cdot t_{r}-1} .
$$


Proof. Let $\underline{w} \in \mathbb{Z}^{r}$ and set $L_{I}:=\left\{\left(a_{1}, \ldots, a_{r}\right) \in K^{r} \mid a_{i}=0\right.$ for $\left.i \in I\right\}$. Then

$$
\begin{aligned}
\chi_{g}\left(\mathbb{P} F_{\underline{v}}^{\mathfrak{b}}\right)= & \chi_{g}\left(\mathbb{P} J^{\mathfrak{b}}(\underline{v}) / J^{\mathfrak{b}}(\underline{v}+\underline{1})\right)-\chi_{g}\left(\bigcup_{i=1}^{r} \mathbb{P}\left(J^{\mathfrak{b}}(\underline{v}) / J^{\mathfrak{b}}(\underline{v}+\underline{1}) \cap L_{\{i\}}\right)\right) \\
= & \chi_{g}\left(\mathbb{P} J^{\mathfrak{b}}(\underline{v}) / J^{\mathfrak{b}}(\underline{v}+\underline{1})\right)-\sum_{\substack{I \subset I_{0} \\
I \neq \varnothing}}(-1)^{\sharp I-1} \chi_{g}\left(\mathbb{P}\left(J^{\mathfrak{b}}(\underline{v}) / J^{\mathfrak{b}}(\underline{v}+\underline{1}) \cap L_{I}\right)\right) \\
= & \sum_{I \subset I_{0}}(-1)^{\sharp I} \chi_{g}\left(\mathbb{P}\left(J^{\mathfrak{b}}(\underline{v}) / J^{\mathfrak{b}}(\underline{v}+\underline{1}) \cap L_{I}\right)\right) \\
= & \sum_{I \subset I_{0}}(-1)^{\sharp I}\left[\mathbb{P}\left(J^{\mathfrak{b}}(\underline{v}) / J^{\mathfrak{b}}(\underline{v}+\underline{1}) \cap L_{I}\right)\right] \\
= & \sum_{I \subset I_{0}}(-1)^{\sharp I}\left[\mathbb{P}\left(J^{\mathfrak{b}}\left(\underline{v}+\underline{1}_{I}\right) / J^{\mathfrak{b}}(\underline{v}+\underline{1})\right)\right] .
\end{aligned}
$$

Therefore

$$
\begin{aligned}
\left(t_{1} \cdot \ldots \cdot t_{r}-1\right) \chi_{g}\left(\mathbb{P} F_{\underline{v}}^{\mathfrak{b}}\right) & =\sum_{\underline{v} \in \mathbb{Z}^{r}} \sum_{I \subset I_{0}}(-1)^{\sharp I}\left[\mathbb{P}\left(J^{\mathfrak{b}}\left(\underline{v}+\underline{1}_{I}-\underline{1}\right) / J^{\mathfrak{b}}(\underline{v})\right)\right] \cdot \underline{t}^{\underline{v}}- \\
& -\sum_{\underline{v} \in \mathbb{Z}^{r}} \sum_{I \subset I_{0}}(-1)^{\sharp I}\left[\mathbb{P}\left(J^{\mathfrak{b}}\left(\underline{v}+\underline{1}_{I}\right) / J^{\mathfrak{b}}(\underline{v}+\underline{1})\right)\right] \cdot \underline{t}^{\underline{v}} \\
& =\sum_{\underline{v} \in \mathbb{Z}^{r}} \sum_{I \subset I_{0}}(-1)^{\sharp I}\left[\mathbb{P}\left(J^{\mathfrak{b}}\left(\underline{v}+\underline{1}_{I}-\underline{1}\right) / J^{\mathfrak{b}}\left(\underline{v}+\underline{1}_{I}\right)\right)\right] \cdot \underline{t}^{\underline{v}} .
\end{aligned}
$$

The coefficient of $\underline{t} \underline{v}$ in the polynomial

$$
\left(\sum_{\underline{v} \in \mathbb{Z}^{r}}\left[\mathbb{P}\left(J^{\mathfrak{b}}(\underline{v}) / J^{\mathfrak{b}}(\underline{v}+\underline{1})\right)\right] \cdot \underline{t^{\underline{v}}}\right) \cdot \prod_{i=1}^{r}\left(t_{i}-1\right)
$$

is equal to

$$
\sum_{I \subset I_{0}}(-1)^{\sharp I}\left[\mathbb{P}\left(J^{\mathfrak{b}}\left(\underline{v}-\underline{1}+\underline{1}_{I}\right) / J^{\mathfrak{b}}\left(\underline{v}+\underline{1}_{I}\right)\right)\right],
$$

and the latter formula coincides with $(*)$.

(5.6) Remark: Since $\mathbb{L}-1$ is invertible in $K_{0}\left(\boldsymbol{\nu}_{k}\right)_{(\mathbb{L})}$, the extended generalised value ideal Poincaré series can be rewritten as

$$
\widehat{P}_{g}(\mathfrak{b}, \underline{t}, \mathbb{L})=\frac{\prod_{i=1}^{r}\left(t_{i}-1\right)}{t_{1} \cdot \ldots \cdot t_{r}-1} \cdot \sum_{\underline{v} \in \mathbb{Z}^{r}} \frac{\mathbb{L}^{c^{\mathfrak{b}}(\underline{v})}-1}{\mathbb{L}-1} \cdot \underline{t}^{\underline{v}} .
$$


We generalise this functional equation by using the following result:

(5.7) Proposition: For every $\underline{v} \in \mathbb{Z}^{r}$, we have

$$
c^{\mathfrak{b}^{*}}(\underline{v})=d-c^{\mathfrak{b}}\left(\gamma^{\mathfrak{b}}-\underline{v}-\underline{1}\right) .
$$

Proof. By means of Proposition (4.16) we deduce the equality:

$$
\begin{aligned}
c^{\mathfrak{b}^{*}}(\underline{v})= & \operatorname{deg}\left(J^{\mathfrak{b}^{*}}(\underline{v})\right)-\operatorname{deg}\left(J^{\mathfrak{b}^{*}}(\underline{v}+\underline{1})\right) \\
= & \operatorname{dim}(\mathfrak{b} / \mathfrak{b}: \overline{\mathcal{O}})-\underline{v} \cdot \underline{d}+\operatorname{deg}\left(J^{\mathfrak{b}}\left(\gamma^{\mathfrak{b}}-\underline{v}\right)\right) \\
& -\operatorname{dim}(\mathfrak{b} / \mathfrak{b}: \overline{\mathcal{O}})-(\underline{v}+\underline{1}) \cdot \underline{d}-\operatorname{deg}\left(J^{\mathfrak{b}}\left(\gamma^{\mathfrak{b}}-\underline{v}-\underline{1}\right)\right) \\
= & d-c^{\mathfrak{b}}\left(\gamma^{\mathfrak{b}}-\underline{v}-\underline{1}\right) .
\end{aligned}
$$

We want to describe functional equations for the series $\widehat{L}_{g}(\mathfrak{b}, \underline{t}, \mathbb{L})$ and the Poincaré series $\widehat{P}_{g}(\mathfrak{b}, \underline{t}, \mathbb{L})$.

(5.8) Theorem:

$$
\begin{gathered}
\underline{t}^{\gamma^{\mathfrak{b}}-1} \widehat{L}_{g}\left(\mathfrak{b}, \underline{t}^{-1}, \mathbb{L}\right)=-\mathbb{L}^{d-1} \widehat{L}_{g}\left(\mathfrak{b}^{*}, \underline{t}, \mathbb{L}^{-1}\right) . \\
\underline{t}^{\gamma^{\mathfrak{b}}-\underline{1}} \widehat{P}_{g}\left(\mathfrak{b}, \underline{t}^{-1}, \mathbb{L}\right)=(-1)^{r} \mathbb{L}^{d-1} \widehat{P}_{g}\left(\mathfrak{b}^{*}, \underline{t}, \mathbb{L}^{-1}\right) .
\end{gathered}
$$

Proof. By Remark (5.6) we have $\widehat{L}_{g}(\mathfrak{b}, \underline{t}, \mathbb{L})=\sum_{\underline{v} \in \mathbb{Z}^{r}} \frac{\mathbb{L}^{c(\underline{v})}-1}{\mathbb{L}-1} \cdot \underline{\underline{v}} \underline{\text {. }}$. It suffices to take $\widetilde{L}_{g}(\mathfrak{b}, \underline{t}, \mathbb{L})=\sum_{\underline{v} \in \mathbb{Z}^{r}} \frac{\mathbb{L}^{c(\underline{v})}}{\mathbb{L}-1} \cdot \underline{t} \underline{\underline{v}}$. By Proposition (5.7) it holds $c^{\mathfrak{b}^{*}}(\underline{v})+c^{\mathfrak{b}}(\gamma-\underline{v}-\underline{1})=d$ and we have

$$
\underline{t}^{\gamma^{*}-\underline{1}} \cdot \widetilde{L}_{g}\left(\mathfrak{b}, \underline{t}^{-1}, \mathbb{L}\right)=\mathbb{L}^{d} \cdot \sum_{\underline{v} \in \mathbb{Z}^{r}} \frac{\mathbb{L}^{-c^{\mathfrak{b}^{*}}(\underline{v})}}{\mathbb{L}-1} \cdot \underline{t}^{\underline{v}} .
$$

On the other hand, we have

$$
\begin{aligned}
\widetilde{L}_{g}\left(\mathfrak{b}^{*}, \underline{t}, \mathbb{L}^{-1}\right) & =\sum_{\underline{v} \in \mathbb{Z}^{r}} \frac{\mathbb{L}^{-c^{\mathfrak{b}^{*}}(\underline{v})}}{\mathbb{L}^{-1}-1} \cdot \underline{t}^{\underline{v}} \\
& =-\mathbb{L} \cdot \sum_{\underline{v} \in \mathbb{Z}^{r}} \frac{\mathbb{L}^{-c^{\mathfrak{b}^{*}}(\underline{v})}}{\mathbb{L}-1} \cdot \underline{t}^{\underline{v}} .
\end{aligned}
$$

Hence

$$
-\mathbb{L}^{d-1} \cdot \widetilde{L}_{g}\left(\mathfrak{b}^{*}, \underline{t}, \mathbb{L}^{-1}\right)=\underline{t}^{\gamma^{\mathfrak{b}}-1} \cdot \widetilde{L}_{g}\left(\mathfrak{b}, \underline{t}^{-1}, \mathbb{L}\right) .
$$

Taking now into account that

$$
\widehat{P}_{g}(\mathfrak{b}, \underline{t}, \mathbb{L})=\frac{\left(t_{1}-1\right) \cdot \ldots \cdot\left(t_{r}-1\right)}{t_{1} \cdot \ldots \cdot t_{r}-1} \cdot \widetilde{L}_{g}(\mathfrak{b}, \underline{t}, \mathbb{L})
$$


and the fact that

$$
(-1)^{r-1} \cdot \frac{\left(t_{1}-1\right) \cdot \ldots \cdot\left(t_{r}-1\right)}{t_{1} \cdot \ldots \cdot t_{r}-1}=\frac{\left(1-t_{1}\right) \cdot \ldots \cdot\left(1-t_{r}\right)}{1-t_{1} \cdot \ldots \cdot t_{r}}
$$

we get

$$
\begin{aligned}
\widehat{P}_{g}\left(\mathfrak{b}, \underline{t}^{-1}, \mathbb{L}\right) & =\frac{\left(t_{1}^{-1}-1\right) \cdot \ldots \cdot\left(t_{r}^{-1}-1\right)}{t_{1}^{-1} \cdot \ldots \cdot t_{r}^{-1}-1} \cdot \widetilde{L}_{g}\left(\mathfrak{b}, \underline{t}^{-1}, \mathbb{L}\right) \\
& =\frac{\left(1-t_{1}\right) \cdot \ldots \cdot\left(1-t_{r}\right)}{1-t_{1} \cdot \ldots \cdot t_{r}} \cdot \widetilde{L}_{g}\left(\mathfrak{b}, \underline{t}^{-1}, \mathbb{L}\right) \\
& =(-1)^{r-1} \cdot \frac{\left(t_{1}-1\right) \cdot \ldots \cdot\left(t_{r}-1\right)}{t_{1} \cdot \ldots \cdot t_{r}-1} \cdot \widetilde{L}_{g}\left(\mathfrak{b}, \underline{t}^{-1}, \mathbb{L}\right) .
\end{aligned}
$$

Since

$$
\widehat{P}_{g}\left(\mathfrak{b}^{*}, \underline{t}, \mathbb{L}^{-1}\right)=\frac{\left(t_{1}-1\right) \cdot \ldots \cdot\left(t_{r}-1\right)}{t_{1} \cdot \ldots \cdot t_{r}-1} \cdot \widetilde{L}_{g}\left(\mathfrak{b}^{*}, \underline{t}, \mathbb{L}^{-1}\right),
$$

we have

$$
\begin{aligned}
\underline{t}^{\gamma^{*}-1} \cdot \widehat{P}_{g}\left(\mathfrak{b}, \underline{t}^{-1}, \mathbb{L}\right) & =(-1)^{r-1} \cdot \frac{\left(t_{1}-1\right) \cdot \ldots \cdot\left(t_{r}-1\right)}{t_{1} \cdot \ldots \cdot t_{r}-1} \cdot \underline{t}^{\gamma^{*}-1} \cdot \widetilde{L}_{g}\left(\mathfrak{b}, \underline{t}^{-1}, \mathbb{L}\right) \\
& =(-1)^{r-1} \cdot \frac{\left(t_{1}-1\right) \cdot \ldots \cdot\left(t_{r}-1\right)}{t_{1} \cdot \ldots \cdot t_{r}-1} \cdot(-\mathbb{L})^{d-1} \cdot \widetilde{L}_{g}\left(\mathfrak{b}^{*}, \underline{t}, \mathbb{L}^{-1}\right) \\
& =(-1)^{r} \cdot \frac{\left(t_{1}-1\right) \cdot \ldots \cdot\left(t_{r}-1\right)}{t_{1} \cdot \ldots \cdot t_{r}-1} \cdot \mathbb{L}^{d-1} \cdot \widetilde{L}_{g}\left(\mathfrak{b}^{*}, \underline{t}, \mathbb{L}^{-1}\right) \\
& =(-1)^{r} \cdot \mathbb{L}^{d-1} \cdot \widehat{P}_{g}\left(\mathfrak{b}^{*}, \underline{t}, \mathbb{L}^{-1}\right),
\end{aligned}
$$

and we are done.

\section{REFERENCES}

[1] A. Campillo, F. Delgado, S.M. Gusein-Zade: The Extended Semigroup of a Plane Curve Singularity. Proc. Steklov Institute of Math. 221, 139-156 (1998).

[2] A. Campillo, F. Delgado, S.M. Gusein-Zade: On the monodromy of a plane curve singularity and the Poincaré series of its rings of functions. Functional Analisis and its Applications 33 (1), 56-57 (1999).

[3] A. Campillo, F. Delgado, S.M. Gusein-Zade: Integration with respect to the Euler characteristic over a function space, and the Alexander polynomial of a plane curve singularity. (Russian) Uspekhi Mat. Nauk 55(6)(336), 127-128 (2000); translation in Russian Math. Surveys 55(6), 1148-1149 (2000).

[4] A. Campillo, F. Delgado, S.M. Gusein-Zade: Multi-index filtrations and motivic Poincaré series. Monatshefte für Mathematik 150, 193-209 (2007).

[5] A. Campillo, F. Delgado and K. Kiyek, Gorenstein property and symmetry for one-dimensional local Cohen-Macaulay rings, Manuscripta Math., 83, 405-423 (1994).

[6] F. Delgado de la Mata: Gorenstein curves and symmetry of the semigroup of values. Manuscripta Math., 61, 285-296 (1988). 
[7] F. Delgado, J.J. Moyano: On the relation between the generalized Poincaré series and the Stöhr Zeta function. Proc. of the Amer. Math. Soc. 137(1), $51-59$ (2009).

[8] J. Herzog, E. Kunz: Der kanonische Modul eines Cohen-Macaulay Rings. Springer Lecture Notes in Math. 238 (1971).

[9] K.Kiyek, J.L.Vicente: Resolution of Curve and Surface Singularities in Characteristic Zero. Kluwer, Dordrecht, 2004.

[10] E. Kunz: The value-semigroup of a one dimensional Gorenstein ring. Proc. Amer. Math. Soc. 25, 748-751 (1970).

[11] J.J. Moyano-Fernández, W.A. Zúñiga: Motivic zeta functions for curve singularities. Nagoya Math. Journal 198, 47-75 (2010).

[12] K.O. Stöhr: Local and Global Zeta-Functions of Singular Algebraic Curves. Journal of Number Theory 71, 172-202 (1998).

[13] K.O. Stöhr: Multi-variable Poincaré Series of Algebraic Curve Singularities over Finite Fields. Mathematische Zeitschrift 262(4), 849-866 (2009).

Institut für Mathematik, Universität Osnabrück. Albrechtstrasse 28A, D-49076 Osnabrück, Germany

E-mail address: jmoyanof@uni-osnabrueck.de 